\title{
Metal-Free Bond Activation by Carboranyl Diphosphines
}

\section{Supporting Information}

Gayathri B. Gange ${ }^{\mathrm{a}}$, Amanda L. Humphries ${ }^{\mathrm{a}}$, Dmitry E. Royzman ${ }^{\mathrm{a}}$, Mark D. Smith ${ }^{\mathrm{a}}$, Dmitry V. Peryshkov**

a). Department of Chemistry and Biochemistry, University of South Carolina 631 Sumter St., Columbia,South Carolina 29208, United States.

Experimental details

S-02

NMR spectra of the carboranyl phosphines 1-7

$\mathrm{S}-05$

Cyclic voltammograms of 1-4

S-30

$\mathrm{X}$-ray single crystal structure determination details

S-34

Figure S-30. The displacement ellipsoid plot of 6

$\mathrm{S}-42$

Computational details

S-45

References

S-48 


\section{Experimental section}

All synthetic manipulations, unless stated otherwise, were carried out either in a nitrogen-filled VAC glovebox or on a dual manifold Schlenk-style vacuum line. The solvents were sparged with nitrogen, passed through activated alumina, and stored over activated $4 \AA$ Linde-type molecular sieves. Benzene- $d^{6}$, THF- $d^{8}$ and dichloromethane- $d^{2}$ were degassed and stored over activated $4 \AA$ Linde-type molecular sieves. NMR spectra were recorded using Varian spectrometers at $400\left({ }^{1} \mathrm{H}\right)$, $100\left({ }^{13} \mathrm{C}\right), 128\left({ }^{11} \mathrm{~B}\right) \mathrm{MHz}$, reported in $\delta$ (parts per million) and referenced to the residual ${ }^{1} \mathrm{H} /{ }^{13} \mathrm{C}$ signals of the deuterated solvent or an external $\mathrm{BF}_{3}\left(\mathrm{Et}_{2} \mathrm{O}\right)\left({ }^{11} \mathrm{~B}(\delta): 0.0 \mathrm{ppm}\right)$ standard. $J$ values are given in $\mathrm{Hz}$.

$\left(\mathrm{P}^{\mathrm{i}} \mathrm{Pr}_{2}\right)_{2}\left(\mathrm{C}_{2} \mathrm{~B}_{10} \mathrm{H}_{10}\right)(3)$ was prepared according to the previously reported literature procedure. ${ }^{1}$ Ortho-carborane $\mathrm{C}_{2} \mathrm{~B}_{10} \mathrm{H}_{12}$ (Boron Specialties), $n$-Butyllithium solution ( ${ }^{\mathrm{B}} \mathrm{BuLi}, 2.5 \mathrm{M}$ solution in hexanes, Sigma-Aldrich), and the other chemicals were used as received.

\section{Synthesis of 1-P'Bu $\mathrm{Bu}_{2}-2-\mathrm{P}^{\mathrm{i}} \mathrm{Pr}_{2}-\mathrm{C}_{2} \mathrm{~B}_{10} \mathrm{H}_{10}$ (1)}

In a glovebox, ortho-carborane $(0.300 \mathrm{~g}, 2.080 \mathrm{mmol})$ was dissolved in THF and ${ }^{\mathrm{n}} \mathrm{BuLi}$ solution in hexanes $(0.91 \mathrm{~mL}, 2.287 \mathrm{mmol}, 1.1$ equiv.) was added dropwise at room temperature. The mixture was stirred for 1 hour. The sample was dried under vacuum and washed with $5 \mathrm{~mL}$ of hexanes. The residue was dissolved in THF $(3 \mathrm{~mL})$ and a portion of ${ }^{\mathrm{t}} \mathrm{Bu}_{2} \mathrm{PCl}(0.413 \mathrm{~g}, 2.287 \mathrm{mmol}, 1.1$ equiv.) was added. The solution was stirred for 4 hours at room temperature. The reaction mixture was dried under vacuum and extracted in $10 \mathrm{~mL}$ of hexanes. The solution was dried under vacuum and the residue was dissolved in $3 \mathrm{~mL}$ of THF, and ${ }^{\mathrm{n}} \mathrm{BuLi}$ solution in hexanes $(0.91 \mathrm{~mL}, 2.287$ mmol, 1.1 equiv.) was added dropwise at room temperature. The mixture was stirred for 1 hour. The sample was dried under vacuum and washed with $5 \mathrm{~mL}$ of hexanes. The residue was dissolved in THF ( $3 \mathrm{~mL})$, and a portion of ${ }^{\mathrm{i}} \mathrm{Pr}_{2} \mathrm{PCl}(0.352 \mathrm{~g}, 2.287 \mathrm{mmol}, 1.1$ equiv.) was added. The mixture was stirred for 4 hours at room temperature. The reaction mixture was dried under vacuum and taken outside of a glovebox. Water was added $(10 \mathrm{~mL})$ and the product was extracted in diethyl ether $(15 \mathrm{~mL} \times 3)$. Ether fractions were combined and dried under vacuum yielding pale yellow solid $(0.654 \mathrm{~g}, 78 \%$ yield).

${ }^{31} \mathbf{P}\left\{{ }^{1} \mathrm{H}\right\}$ NMR $\left(\mathrm{C}_{6} \mathrm{D}_{6}\right): \delta 69.7\left(\mathrm{~d},{ }^{3} J_{\mathrm{P}-\mathrm{P}}=90 \mathrm{~Hz}, 1 \mathrm{P}, P\left(\mathrm{C}\left(\mathrm{CH}_{3}\right)_{3}\right)_{2}\right), 28.1\left(\mathrm{~d},{ }^{3} J_{\mathrm{P}-\mathrm{P}}=90 \mathrm{~Hz}, 1 \mathrm{P}\right.$, $\left.P\left(\mathrm{CH}\left(\mathrm{CH}_{3}\right)_{2}\right)_{2}\right) .{ }^{11} \mathbf{B}\left\{{ }^{1} \mathbf{H}\right\}$ NMR $\left(\mathrm{C}_{6} \mathrm{D}_{6}\right): \delta 1.9(B \mathrm{H}), 0.4(B \mathrm{H}),-5.7(B \mathrm{H}),-8.1(B \mathrm{H}),-9.7(B \mathrm{H}) .{ }^{1} \mathbf{H}$ 
NMR $\left(\mathrm{C}_{6} \mathrm{D}_{6}\right): \delta 2.03\left(\mathrm{~m}, 2 \mathrm{H}, \mathrm{P}\left(\mathrm{CH}\left(\mathrm{CH}_{3}\right)_{2}\right)_{2}\right), 1.27\left(\mathrm{~d},{ }^{3} J_{\mathrm{P}-\mathrm{H}}=12 \mathrm{~Hz}, 18 \mathrm{H}, \mathrm{P}\left(\mathrm{C}\left(\mathrm{CH}_{3}\right)_{3}\right)_{2}\right), 1.15$ to $1.02\left(\mathrm{~m}, 12 \mathrm{H}, \mathrm{P}\left(\mathrm{CH}\left(\mathrm{CH}_{3}\right)_{2}\right)_{2}\right) .{ }^{13} \mathrm{C} \mathbf{N M R}\left(\mathrm{C}_{6} \mathrm{D}_{6}\right): 92.9\left(\mathrm{dd},{ }^{1} J_{\mathrm{C}-\mathrm{P}}=83 \mathrm{~Hz},{ }^{2} J_{\mathrm{C}-\mathrm{P}}=17 \mathrm{~Hz}, \mathrm{CP}\right.$ (orthocarborane)), $92.6\left(\mathrm{dd},{ }^{1} J_{\mathrm{C}-\mathrm{P}}=109 \mathrm{~Hz},{ }^{2} J_{\mathrm{C}-\mathrm{P}}=13 \mathrm{~Hz}, C \mathrm{P}\right.$ (ortho-carborane)), $39.3\left(\mathrm{~d},{ }^{1} J_{\mathrm{C}-\mathrm{P}}=40 \mathrm{~Hz}\right.$, $\left.\mathrm{P}\left(\mathrm{C}\left(\mathrm{CH}_{3}\right)_{3}\right)_{2}\right), 32.2\left(\mathrm{~d},{ }^{2} J_{\mathrm{C}-\mathrm{P}}=14 \mathrm{~Hz}, \mathrm{P}\left(\mathrm{C}\left(\mathrm{CH}_{3}\right)_{3}\right)_{2}\right), 26.7\left(\mathrm{~d},{ }^{1} J_{\mathrm{C}-\mathrm{P}}=26 \mathrm{~Hz}, \mathrm{P}\left(\mathrm{CH}\left(\mathrm{CH}_{3}\right)_{2}\right)_{2}\right), 22.6(\mathrm{~d}$, $\left.{ }^{2} J_{\mathrm{C}-\mathrm{P}}=27 \mathrm{~Hz}, \mathrm{P}\left(\mathrm{CH}\left(\mathrm{CH}_{3}\right)_{2}\right)_{2}\right), 19.0\left(\mathrm{~d},{ }^{2} J_{\mathrm{C}-\mathrm{P}}=8 \mathrm{~Hz}, \mathrm{P}\left(\mathrm{CH}\left(\mathrm{CH}_{3}\right)_{2}\right)_{2}\right)$. Anal. Calcd. for $\mathrm{C}_{16} \mathrm{H}_{42} \mathrm{~B}_{10} \mathrm{P}_{2}$ : C, 47.50; H, 10.46. Found: C, 47.52; H, 10.56.

\section{Synthesis of $\mathrm{Na}_{2}\left[\mathrm{P}^{t} \mathrm{Bu}_{2}-\mathrm{P}^{\mathrm{i}} \mathrm{Pr}_{2}-\left(\mathrm{C}_{2} \mathrm{~B}_{10} \mathrm{H}_{10}\right)\right]$ (2)}

A portion of compound $1(0.030 \mathrm{~g}, 0.074 \mathrm{mmol})$ was dissolved in $1 \mathrm{~mL}$ of THF and sodium metal was added $(0.009 \mathrm{~g}, 0.371 \mathrm{mmol}, 5$ equiv.). The reaction mixture was stirred for 4 hours at room temperature. Volatiles were removed under vacuum. Yield: $0.044 \mathrm{~g}$.

${ }^{31} \mathbf{P}\left\{{ }^{1} \mathbf{H}\right\}$ NMR $\left.\left(\mathrm{THF}-\mathrm{d}_{8}\right): \delta 65.6\left(\mathrm{~s}, 1 \mathrm{P}, P\left(\mathrm{C}_{(\mathrm{CH}}\right)_{3}\right)_{2}\right), 39.7\left(\mathrm{~s}, 1 \mathrm{P}, P\left(\mathrm{CH}\left(\mathrm{CH}_{3}\right)_{2}\right)_{2}\right) .{ }^{11} \mathbf{B}\left\{{ }^{1} \mathbf{H}\right\}$ NMR (THF-d $\left.\mathrm{d}_{8}\right): \delta 21.8(B \mathrm{H}),-1.1(B \mathrm{H}),-2.1(B \mathrm{H}),-18.3(B \mathrm{H}),-21.8(B \mathrm{H}) .{ }^{1} \mathbf{H}$ NMR (THF-d 8$): \delta 2.06$ (sp, 2H, $\left.\mathrm{P}\left(\mathrm{CH}\left(\mathrm{CH}_{3}\right)_{2}\right)_{2}\right), 1.27\left(\mathrm{~d},{ }^{3} \mathrm{~J}_{\mathrm{P}-\mathrm{H}}=10 \mathrm{~Hz}, 18 \mathrm{H}, \mathrm{P}\left(\mathrm{C}\left(\mathrm{CH}_{3}\right)_{3}\right)_{2}\right), 1.20$ to $1.08(\mathrm{~m}, 12 \mathrm{H}$, $\left.\mathrm{P}\left(\mathrm{CH}\left(\mathrm{CH}_{3}\right)_{2}\right)_{2}\right) .{ }^{13} \mathrm{C}$ NMR $\left(\mathrm{THF}-\mathrm{d}_{8}\right)$ : 90.6 (s, CP (ortho-carborane)), 86.8 (s, CP (orthocarborane)), $34.4\left(\mathrm{~d},{ }^{1} J_{\mathrm{P}-\mathrm{C}}=20 \mathrm{~Hz}, \mathrm{P}\left(\mathrm{C}\left(\mathrm{CH}_{3}\right)_{3}\right)_{2}\right), 31.5\left(\mathrm{~d},{ }^{2} J_{\mathrm{P}-\mathrm{C}}=12 \mathrm{~Hz}, \mathrm{P}\left(\mathrm{C}\left(\mathrm{CH}_{3}\right)_{3}\right)_{2}\right), 28.0\left(\mathrm{~d},{ }^{1} J_{\mathrm{P}-}\right.$ $\left.\mathrm{C}=56 \mathrm{~Hz}, \mathrm{P}\left(\mathrm{CH}\left(\mathrm{CH}_{3}\right)_{2}\right)_{2}\right), 22.2\left(\mathrm{~d},{ }^{2} J_{\mathrm{P}-\mathrm{C}}=18 \mathrm{~Hz}, \mathrm{P}\left(\mathrm{CH}\left(\mathrm{CH}_{3}\right)_{2}\right)_{2}\right), 20.2 \quad\left(\mathrm{~d},{ }^{2} J_{\mathrm{P}-\mathrm{C}}=10 \mathrm{~Hz}\right.$, $\left.\mathrm{P}\left(\mathrm{CH}\left(\mathrm{CH}_{3}\right)_{2}\right)_{2}\right)$.

\section{Synthesis of $\mathrm{Na}_{2}(\mathrm{THF})_{3}\left[\left(\mathrm{PiPr}_{2}\right)_{2}-\left(\mathrm{C}_{2} \mathrm{~B}_{10} \mathrm{H}_{10}\right)\right]$ (4)}

A portion of compound $3(0.030 \mathrm{~g}, 0.078 \mathrm{mmol})$ was dissolved in $1 \mathrm{~mL}$ of THF and sodium metal was added $(0.009 \mathrm{~g}, 0.390 \mathrm{mmol}, 5$ equiv.). The reaction mixture was stirred for 2 hours at room temperature. Volatiles were removed under vacuum. Single crystals were grown from THF/toluene solvent mixture. Yield: $0.035 \mathrm{~g}$

${ }^{31} \mathbf{P}\left\{{ }^{1} \mathbf{H}\right\}$ NMR $\left(\right.$ THF-d $\left.\mathrm{d}_{8}\right): \delta 34.3\left(\mathrm{~s}, P\left(\mathrm{CH}\left(\mathrm{CH}_{3}\right)_{2}\right)_{2}\right) .{ }^{11} \mathbf{B}\left\{{ }^{1} \mathbf{H}\right\}$ NMR $\left(\mathrm{THF}-\mathrm{d}_{8}\right): \delta 18.8(B \mathrm{H}),-3.2$ $(B \mathrm{H}),-9.6(B \mathrm{H}),-13.1(B \mathrm{H}),-18.7(B \mathrm{H}),-22.2(B \mathrm{H}),-26.3(B \mathrm{H}) .{ }^{1} \mathrm{H}$ NMR (THF-d 8$): \delta 2.07(\mathrm{sp}$, 4H, $\left.\mathrm{P}\left(\mathrm{CH}\left(\mathrm{CH}_{3}\right)_{2}\right)_{2}\right), 1.15$ (m, 24H, $\left.\mathrm{P}\left(\mathrm{CH}\left(\mathrm{CH}_{3}\right)_{2}\right)_{2}\right) .{ }^{13} \mathrm{C}$ NMR $\left(\mathrm{THF}-\mathrm{d}_{8}\right): 90.6$ (s, CP (orthocarborane $)), 29.0\left(\mathrm{~d},{ }^{1} J_{\mathrm{P}-\mathrm{C}}=13 \mathrm{~Hz}, \mathrm{P}\left(\mathrm{CH}\left(\mathrm{CH}_{3}\right)_{2}\right)_{2}\right), 21.9\left(\mathrm{~d},{ }^{2} J_{\mathrm{P}-\mathrm{C}}=17 \mathrm{~Hz}, \mathrm{P}\left(\mathrm{CH}\left(\mathrm{CH}_{3}\right)_{2}\right)_{2}\right), 19.9(\mathrm{~d}$, $\left.{ }^{2} J_{\mathrm{P}-\mathrm{C}}=10 \mathrm{~Hz}, \mathrm{P}\left(\mathrm{CH}\left(\mathrm{CH}_{3}\right)_{2}\right)_{2}\right)$.

Synthesis of $\mathbf{P}(\mathrm{H})^{\mathrm{t}} \mathrm{Bu}_{2}-\mathrm{P}(\mathrm{H})^{\mathrm{i}} \mathrm{Pr}_{2}-\left(\mathrm{C}_{2} \mathrm{~B}_{10} \mathrm{H}_{10}\right)(5)$ 
A portion of tributylstannane $(0.076 \mathrm{~g}, 0.260 \mathrm{mmol}, 2.1$ equiv. $)$ was added to a solution of compound 1 ( $0.050 \mathrm{~g}, 0.124 \mathrm{mmol})$ in $1 \mathrm{~mL}$ of benzene. The solution was heated at $70{ }^{\circ} \mathrm{C}$ for 2 days and the resulting precipitate was separated and dried completely under vacuum. The reaction mixture was washed with hexanes $(2 \mathrm{~mL} \times 3)$ to afford a white color solid product. Yield: $0.047 \mathrm{~g}$ $(97 \%)$

${ }^{31} \mathbf{P}\left\{{ }^{1} \mathrm{H}\right\}$ NMR $\left(\mathrm{CD}_{2} \mathrm{Cl}_{2}\right): \delta 60.7\left(\mathrm{~s}, 1 \mathrm{P}, P\left(\mathrm{C}\left(\mathrm{CH}_{3}\right)_{3}\right)_{2}\right), 42.9$ (s, 1P, $P\left(\mathrm{CH}_{\left.\left.\left(\mathrm{CH}_{3}\right)_{2}\right)_{2}\right) .}{ }^{31} \mathbf{P}\right.$ NMR

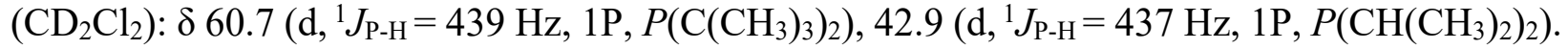
${ }^{11} \mathbf{B}\left\{{ }^{1} \mathbf{H}\right\}$ NMR $\left(\mathrm{CD}_{2} \mathrm{Cl}_{2}\right): \delta 23.6(B \mathrm{H}), 3.2(B \mathrm{H}), 2.7(B \mathrm{H}),-15.8(B \mathrm{H}),-17.2(B \mathrm{H}),-18.8(B \mathrm{H}) .{ }^{1} \mathbf{H}$ NMR $\left(\mathrm{CD}_{2} \mathrm{Cl}_{2}\right): \delta 5.46\left(\mathrm{td},{ }^{1} J_{\mathrm{P}-\mathrm{H}}=437 \mathrm{~Hz},{ }^{3} J_{\mathrm{H}-\mathrm{H}}=3 \mathrm{~Hz}, 1 \mathrm{H}, H-\mathrm{P}\left(\mathrm{CH}\left(\mathrm{CH}_{3}\right)_{2}\right)_{2}\right), 5.22\left(\mathrm{~d},{ }^{1} J_{\mathrm{P}-\mathrm{H}}=\right.$

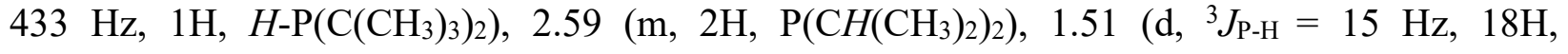
$\left.\mathrm{P}\left(\mathrm{C}\left(\mathrm{CH}_{3}\right)_{3}\right)_{2}\right), 1.39\left(\mathrm{~m}, 12 \mathrm{H}, \mathrm{P}\left(\mathrm{CH}\left(\mathrm{CH}_{3}\right)_{2}\right)_{2}\right) .{ }^{13} \mathbf{C} \mathbf{N M R}\left(\mathrm{CD}_{2} \mathrm{Cl}_{2}\right): \delta 35.8\left(\mathrm{~d},{ }^{1} J_{\mathrm{C}-\mathrm{P}}=41 \mathrm{~Hz}\right.$, $\left.\mathrm{P}\left(\mathrm{C}\left(\mathrm{CH}_{3}\right)_{3}\right)_{2}\right), 28.8\left(\mathrm{~s}, \mathrm{P}\left(\mathrm{C}\left(\mathrm{CH}_{3}\right)_{3}\right)_{2}\right), 23.7\left(\mathrm{~d},{ }^{1} J_{\mathrm{C}-\mathrm{P}}=47 \mathrm{~Hz}, \mathrm{P}\left(\mathrm{CH}\left(\mathrm{CH}_{3}\right)_{2}\right)_{2}\right), 18.4\left(\mathrm{~d},{ }^{2} J_{\mathrm{C}-\mathrm{P}}=2 \mathrm{~Hz}\right.$, $\left.\mathrm{P}\left(\mathrm{CH}\left(\mathrm{CH}_{3}\right)_{2}\right)_{2}\right), 17.6\left(\mathrm{~d},{ }^{2} J_{\mathrm{C}-\mathrm{P}}=2 \mathrm{~Hz}, \mathrm{P}\left(\mathrm{CH}\left(\mathrm{CH}_{3}\right)_{2}\right)_{2}\right)$. Anal. Calcd. for $\mathrm{C}_{16} \mathrm{H}_{44} \mathrm{~B}_{10} \mathrm{P}_{2}: \mathrm{C}, 47.27 ; \mathrm{H}$, 10.91. Found: 47.32; H, 10.67

\section{Synthesis of $\mathrm{P}^{\mathrm{t}} \mathrm{Bu}_{2}(\mathrm{OMe})-\mathrm{PiPr}_{2}(\mathrm{H})-\left(\mathrm{C}_{2} \mathrm{~B}_{10} \mathrm{H}_{10}\right)(7)$.}

A portion of compound $1(0.050 \mathrm{~g}, 0.124 \mathrm{mmol})$ was dissolved in $1 \mathrm{~mL}$ of benzene. Tosylic acid ( $0.004 \mathrm{~g}, 0.025 \mathrm{mmol}, 0.2$ equiv.) and methanol (0.040 g, $1.238 \mathrm{mmol}, 10$ equiv.) were added to the sample. The reaction mixture was stirred for 4 hours at room temperature. The sample was dried under vacuum and washed with hexanes $(2 \mathrm{~mL} \times 3)$ and water $(3 \mathrm{~mL} \times 2)$. Yield: $0.048 \mathrm{~g}$ $(88 \%)$.

${ }^{31} \mathbf{P}\left\{{ }^{1} \mathrm{H}\right\}$ NMR $\left(\mathrm{CD}_{2} \mathrm{Cl}_{2}\right): \delta 96.4\left(\mathrm{~s}, 1 \mathrm{P}, P\left(\mathrm{C}\left(\mathrm{CH}_{3}\right)_{3}\right)_{2}\right), 42.0\left(\mathrm{~s}, 1 \mathrm{P}, \mathrm{H}-P\left(\mathrm{CH}\left(\mathrm{CH}_{3}\right)_{2}\right)_{2}\right) .{ }^{31} \mathbf{P}$ NMR $\left(\mathrm{CD}_{2} \mathrm{Cl}_{2}\right): \delta 96.4\left(\mathrm{~s}, 1 \mathrm{P}, P\left(\mathrm{C}\left(\mathrm{CH}_{3}\right)_{3}\right)_{2}\right), 42.0\left(\mathrm{~d},{ }^{1} \mathrm{~J}_{\mathrm{P}-\mathrm{H}}=435 \mathrm{~Hz}, 1 \mathrm{P}, \mathrm{H}-P\left(\mathrm{CH}\left(\mathrm{CH}_{3}\right)_{2}\right)_{2}\right) .{ }^{1} \mathbf{H} \mathbf{~ N M R}$ $\left(\mathrm{CD}_{2} \mathrm{Cl}_{2}\right): \delta 5.43\left(\mathrm{td},{ }^{1} J_{\mathrm{P}-\mathrm{H}}=437 \mathrm{~Hz},{ }^{3} J_{\mathrm{H}-\mathrm{H}}=3 \mathrm{~Hz}, 1 \mathrm{H}, H-\mathrm{P}\left(\mathrm{CH}\left(\mathrm{CH}_{3}\right)_{2}\right)_{2}\right), 3.81\left(\mathrm{~d},{ }^{3} J_{\mathrm{P}-\mathrm{H}}=10 \mathrm{~Hz}\right.$, $\left.3 \mathrm{H}, \mathrm{OCH}_{3}\right), 2.51-2.59\left(\mathrm{~m}, 2 \mathrm{H}, \mathrm{P}\left(\mathrm{CH}\left(\mathrm{CH}_{3}\right)_{2}\right)_{2}\right), 1.43\left(\mathrm{~d},{ }^{3} \mathrm{~J}_{\mathrm{P}-\mathrm{H}}=15 \mathrm{~Hz}, 18 \mathrm{H}, \mathrm{P}\left(\mathrm{C}\left(\mathrm{CH}_{3}\right)_{3}\right)_{2}\right), 1.34(\mathrm{~m}$, 12H, P(CH(CH 3$\left.\left.)_{2}\right)_{2}\right) .{ }^{11} \mathbf{B}\left\{{ }^{1} \mathbf{H}\right\}$ NMR $\left(\mathrm{CD}_{2} \mathrm{Cl}_{2}\right): \delta 23.6(B \mathrm{H}), 4.7(B \mathrm{H}), 1.7(B \mathrm{H}),-15.9(B \mathrm{H}),-18.6$ $(B \mathrm{H}) .{ }^{13} \mathrm{C}$ NMR $\left(\mathrm{CD}_{2} \mathrm{Cl}_{2}\right): 57.2\left(\mathrm{~d},{ }^{2} J_{\mathrm{C}-\mathrm{P}}=11 \mathrm{~Hz}, \mathrm{OCH}_{3}\right), 39.3\left(\mathrm{~d},{ }^{1} J_{\mathrm{C}-\mathrm{P}}=59 \mathrm{~Hz}, \mathrm{P}\left(C\left(\mathrm{CH}_{3}\right)_{3}\right)_{2}\right), 28.0$ (s, $\left.\mathrm{P}\left(\mathrm{C}\left(\mathrm{CH}_{3}\right)_{3}\right)_{2}\right), 23.7\left(\mathrm{~d},{ }^{1} J_{\mathrm{C}-\mathrm{P}}=47 \mathrm{~Hz}, \mathrm{P}\left(\mathrm{CH}\left(\mathrm{CH}_{3}\right)_{2}\right)_{2}\right), 18.5\left(\mathrm{~d},{ }^{2} J_{\mathrm{C}-\mathrm{P}}=3 \mathrm{~Hz}, \mathrm{P}\left(\mathrm{CH}\left(\mathrm{CH}_{3}\right)_{2}\right)_{2}\right), 17.6$ $\left(\mathrm{d},{ }^{2} J_{\mathrm{C}-\mathrm{P}}=3 \mathrm{~Hz}, \mathrm{P}\left(\mathrm{CH}\left(\mathrm{CH}_{3}\right)_{2}\right)_{2}\right)$. Anal. Calcd. for $\mathrm{C}_{17} \mathrm{H}_{46} \mathrm{~B}_{10} \mathrm{OP}_{2}$ : C, 46.77; H, 10.62. Found: C, 46.72; H, 10.38 . 
NMR spectra of the carboranyl phosphines 1-7
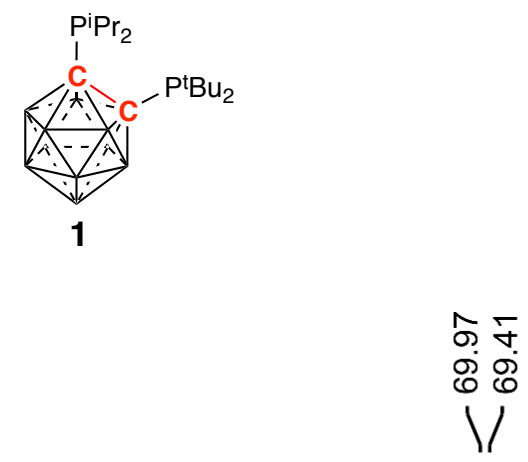

$\hat{m} \infty$

$\stackrel{\infty}{N}$

1

1

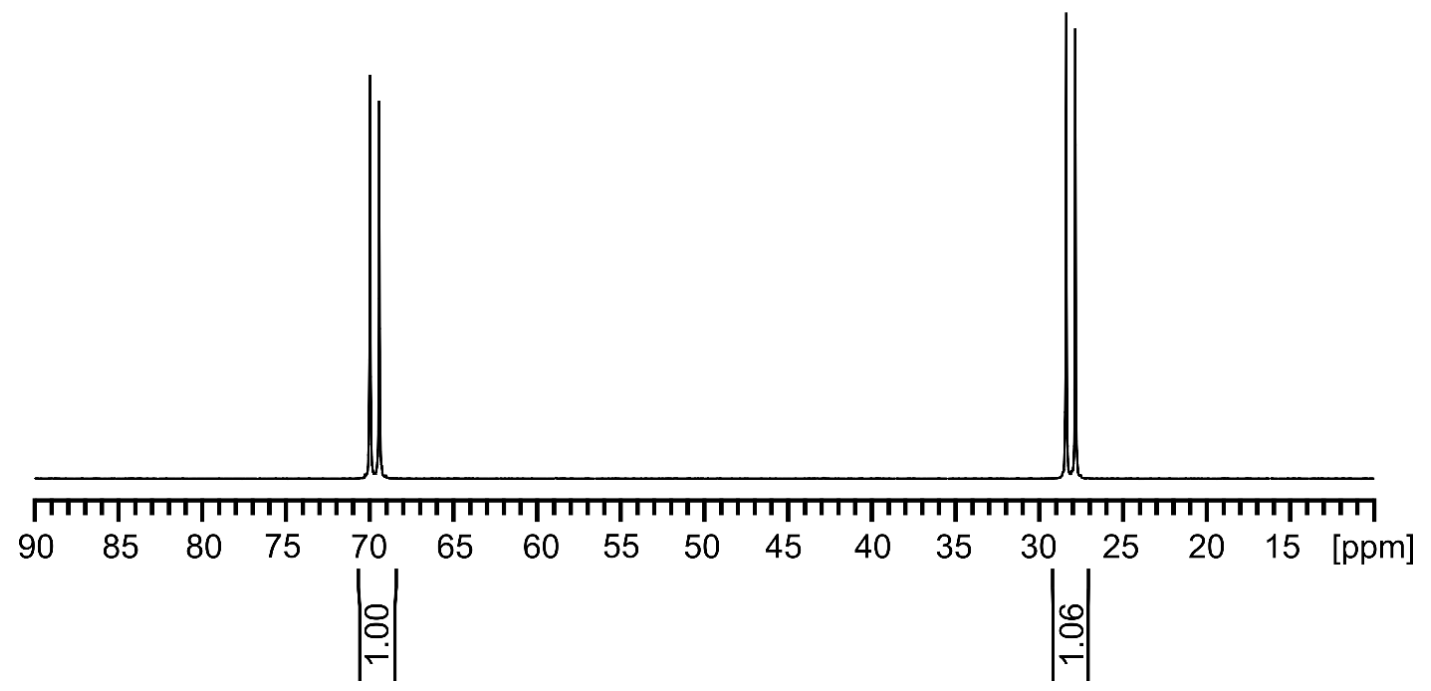

Figure S-1: The ${ }^{31} \mathrm{P}\left\{{ }^{1} \mathrm{H}\right\}$ NMR spectrum of 1-P' $\mathrm{Bu}_{2}-2-\mathrm{P}^{\mathrm{i}} \mathrm{Pr}_{2}-\left(\mathrm{C}_{2} \mathrm{~B}_{10} \mathrm{H}_{10}\right)(\mathbf{1})$.

${ }^{31} \mathbf{P}\left\{{ }^{1} \mathrm{H}\right\}$ NMR $\left(\mathrm{C}_{6} \mathrm{D}_{6}\right): \delta 69.7\left(\mathrm{~d},{ }^{3} J_{\mathrm{P}-\mathrm{P}}=90 \mathrm{~Hz}, 1 \mathrm{P}, P\left(\mathrm{C}\left(\mathrm{CH}_{3}\right)_{3}\right)_{2}\right), 28.1\left(\mathrm{~d},{ }^{3} J_{\mathrm{P}-\mathrm{P}}=90 \mathrm{~Hz}, 1 \mathrm{P}\right.$, $\left.P\left(\mathrm{CH}\left(\mathrm{CH}_{3}\right)_{2}\right)_{2}\right)$. 

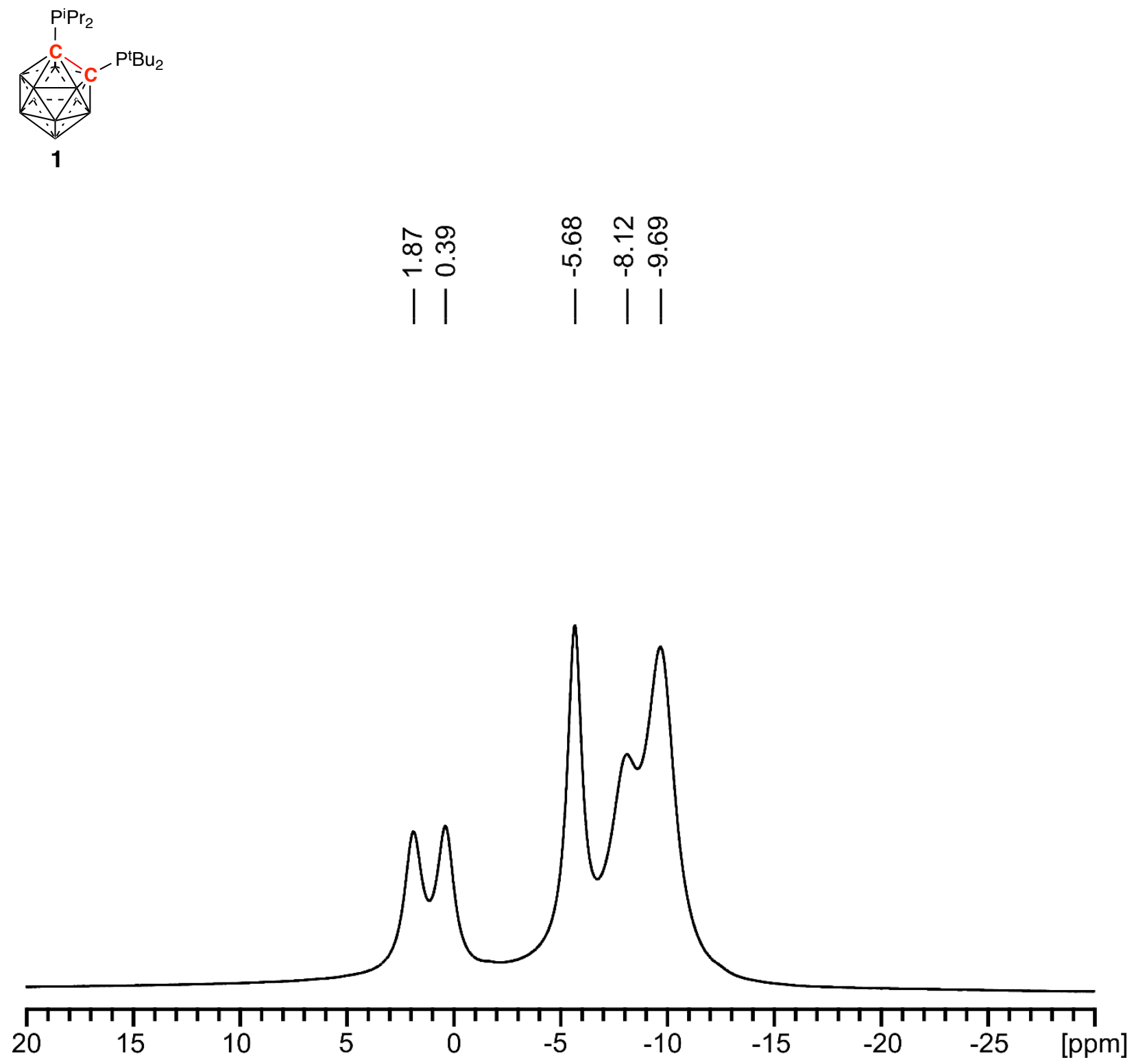

Figure S-2: The ${ }^{11} \mathrm{~B}\left\{{ }^{1} \mathrm{H}\right\}$ NMR spectrum of 1- ${ }^{\mathrm{t}} \mathrm{Bu}_{2}-2-\mathrm{P}^{\mathrm{i}} \mathrm{Pr}_{2}-\left(\mathrm{C}_{2} \mathrm{~B}_{10} \mathrm{H}_{10}\right)(\mathbf{1})$.

${ }^{11} \mathbf{B}\left\{{ }^{1} \mathrm{H}\right\}$ NMR $\left(\mathrm{C}_{6} \mathrm{D}_{6}\right): \delta 1.9(B \mathrm{H}), 0.4(B \mathrm{H}),-5.7(B \mathrm{H}),-8.1(B \mathrm{H}),-9.7(B \mathrm{H})$. 

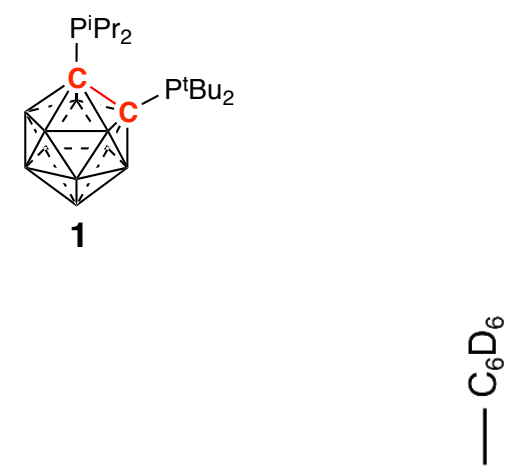

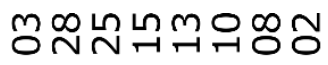

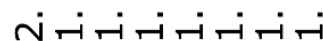

$1 /$

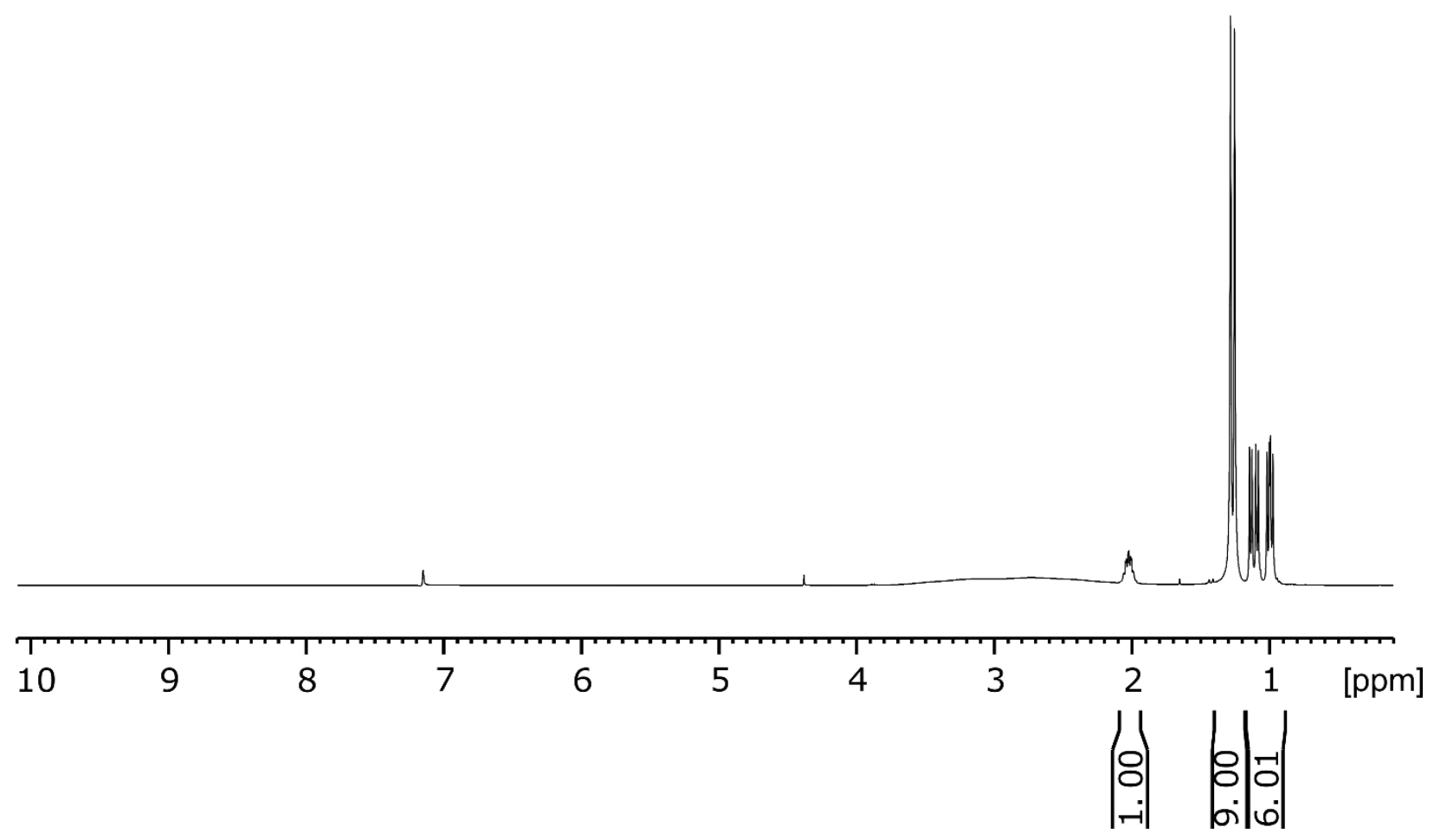

Figure S-3: The ${ }^{1} \mathrm{H}$ NMR spectrum of 1-P'Bu $2-2-\mathrm{P}^{\mathrm{i}} \mathrm{Pr}_{2}-\left(\mathrm{C}_{2} \mathrm{~B}_{10} \mathrm{H}_{10}\right)(\mathbf{1})$.

${ }^{1} \mathrm{H}$ NMR $\left(\mathrm{C}_{6} \mathrm{D}_{6}\right): \delta 2.03\left(\mathrm{~m}, 2 \mathrm{H}, \mathrm{P}\left(\mathrm{CH}\left(\mathrm{CH}_{3}\right)_{2}\right)_{2}\right), 1.27\left(\mathrm{~d}, 18 \mathrm{H}, \mathrm{P}\left(\mathrm{C}\left(\mathrm{CH}_{3}\right)_{3}\right)_{2}\right), 1.15$ to $1.02(\mathrm{~m}, 12 \mathrm{H}$, $\left.\mathrm{P}\left(\mathrm{CH}\left(\mathrm{CH}_{3}\right)_{2}\right)_{2}\right)$. 

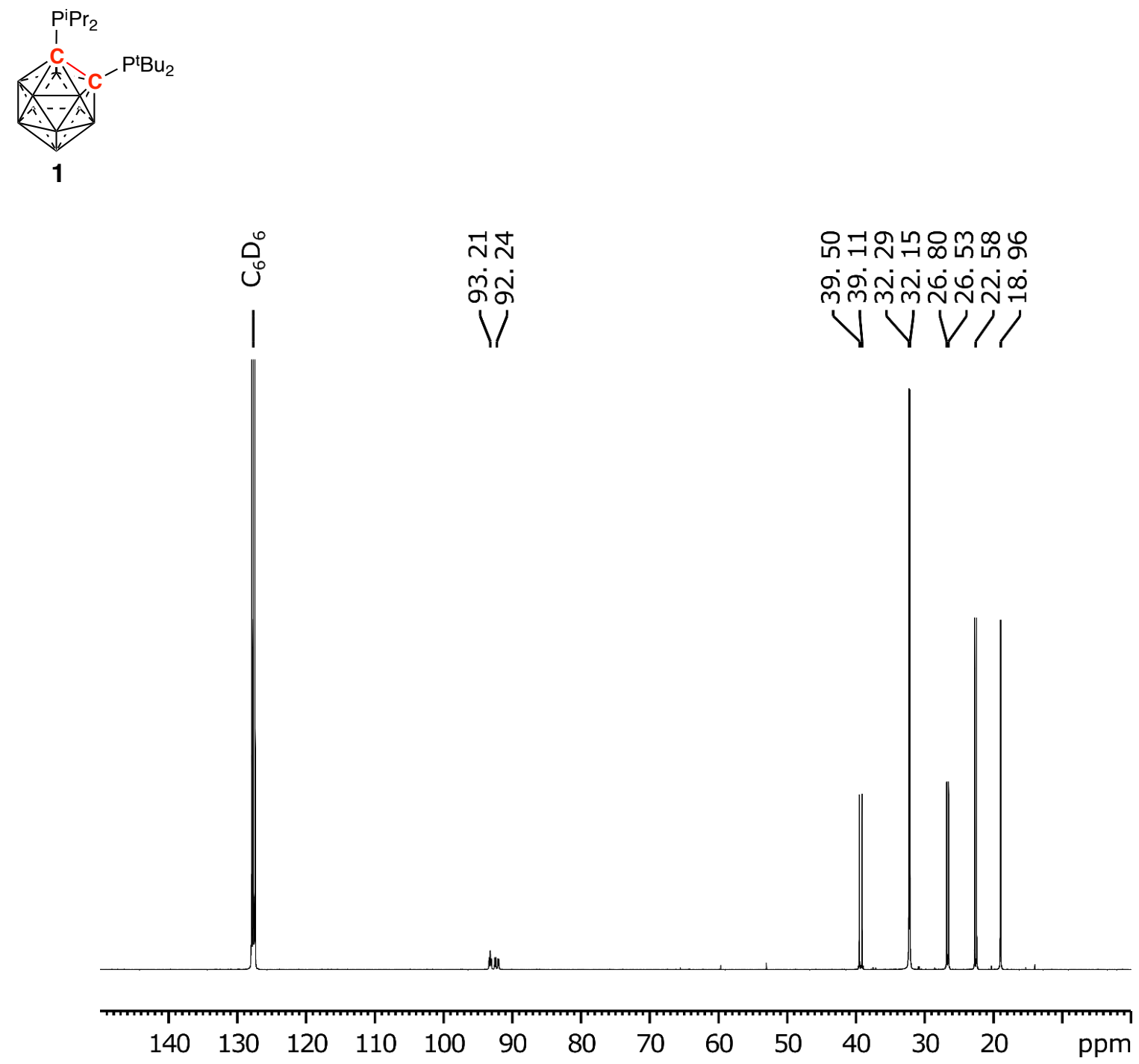

Figure S-4: The ${ }^{13} \mathrm{C}$ NMR spectrum of 1-Pt $\mathrm{Bu}_{2}-2-\mathrm{P}^{\mathrm{i}} \mathrm{Pr}_{2}-\left(\mathrm{C}_{2} \mathrm{~B}_{10} \mathrm{H}_{10}\right)(\mathbf{1})$.

${ }^{13}$ C NMR $\left(\mathrm{C}_{6} \mathrm{D}_{6}\right)$ : $92.9\left(\mathrm{dd},{ }^{1} J_{\mathrm{C}-\mathrm{P}}=83 \mathrm{~Hz},{ }^{2} J_{\mathrm{C}-\mathrm{P}}=17 \mathrm{~Hz}, C \mathrm{P}\right.$ (ortho-carborane)), $92.6\left(\mathrm{dd},{ }^{1} J_{\mathrm{C}-\mathrm{P}}=\right.$ $109 \mathrm{~Hz},{ }^{2} J_{\mathrm{C}-\mathrm{P}}=13 \mathrm{~Hz}, C \mathrm{P}($ ortho-carborane $\left.)\right), 39.3\left(\mathrm{~d},{ }^{1} J_{\mathrm{C}-\mathrm{P}}=40 \mathrm{~Hz}, \mathrm{P}\left(C\left(\mathrm{CH}_{3}\right)_{3}\right)_{2}\right), 32.2\left(\mathrm{~d},{ }^{2} J_{\mathrm{C}-\mathrm{P}}=\right.$ $\left.14 \mathrm{~Hz}, \mathrm{P}\left(\mathrm{C}\left(\mathrm{CH}_{3}\right)_{3}\right)_{2}\right), 26.7\left(\mathrm{~d},{ }^{1} J_{\mathrm{C}-\mathrm{P}}=26 \mathrm{~Hz}, \mathrm{P}\left(\mathrm{CH}\left(\mathrm{CH}_{3}\right)_{2}\right)_{2}\right), 22.6\left(\mathrm{~d},{ }^{2} J_{\mathrm{C}-\mathrm{P}}=27 \mathrm{~Hz}, \mathrm{P}\left(\mathrm{CH}\left(\mathrm{CH}_{3}\right)_{2}\right)_{2}\right)$, $19.0\left(\mathrm{~d},{ }^{2} J_{\mathrm{C}-\mathrm{P}}=8 \mathrm{~Hz}, \mathrm{P}\left(\mathrm{CH}\left(\mathrm{CH}_{3}\right)_{2}\right)_{2}\right)$. 

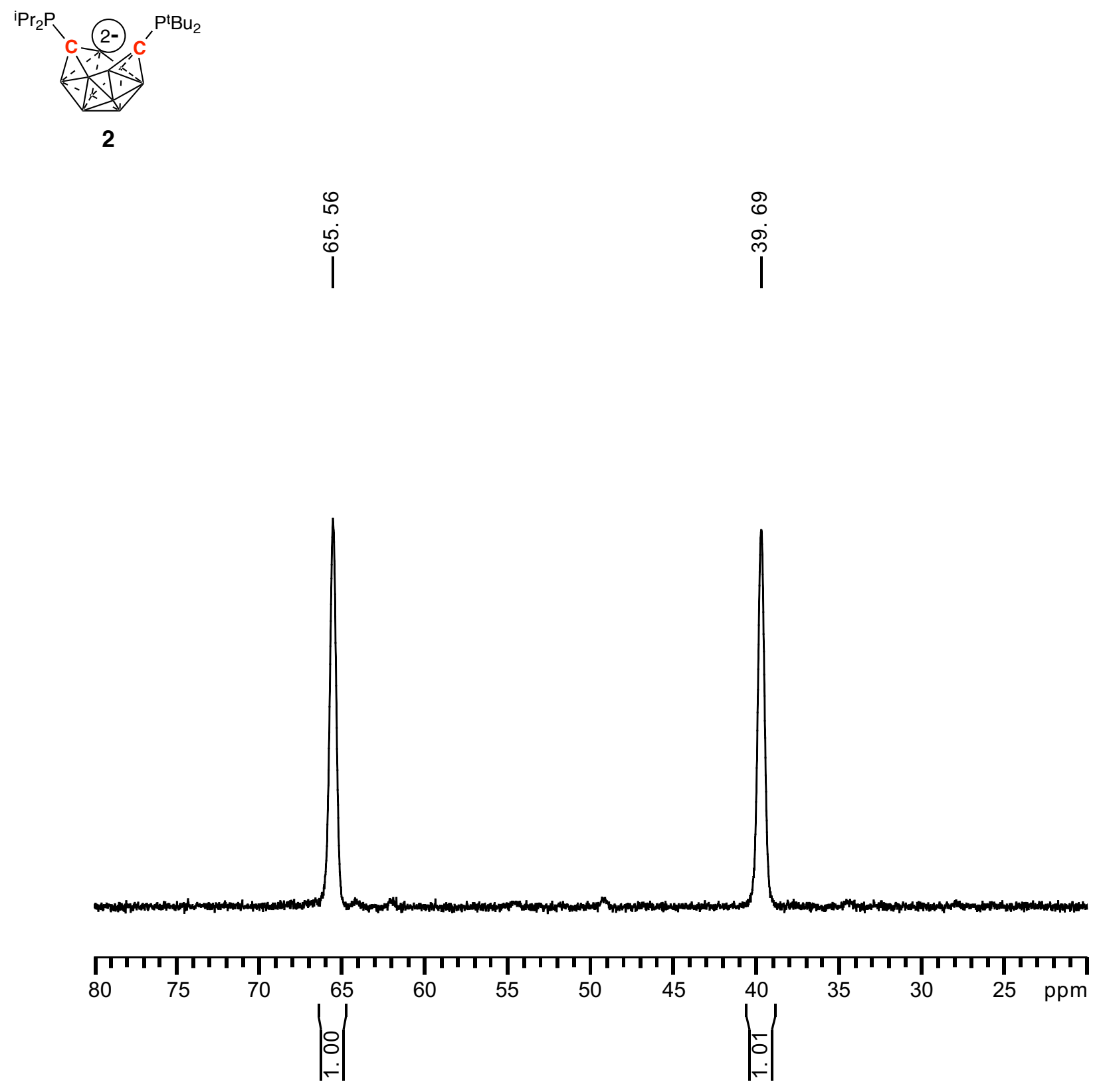

Figure S-5: The ${ }^{31} \mathrm{P}\left\{{ }^{1} \mathrm{H}\right\}$ NMR spectrum of $\mathrm{Na}_{2}\left[\mathrm{P}^{t} \mathrm{Bu}_{2}-\mathrm{P}^{\mathrm{i}} \mathrm{Pr}_{2}-\left(\mathrm{C}_{2} \mathrm{~B}_{10} \mathrm{H}_{10}\right)\right]$ (2).

${ }^{31} \mathbf{P}\left\{{ }^{1} \mathbf{H}\right\}$ NMR $\left.\left(\mathrm{THF}-\mathrm{d}_{8}\right): \delta 65.6\left(\mathrm{~s}, 1 \mathrm{P}, P\left(\mathrm{C}_{(\mathrm{CH}}\right)_{3}\right)_{2}\right), 39.7\left(\mathrm{~s}, 1 \mathrm{P}, P\left(\mathrm{CH}\left(\mathrm{CH}_{3}\right)_{2}\right)_{2}\right)$. 

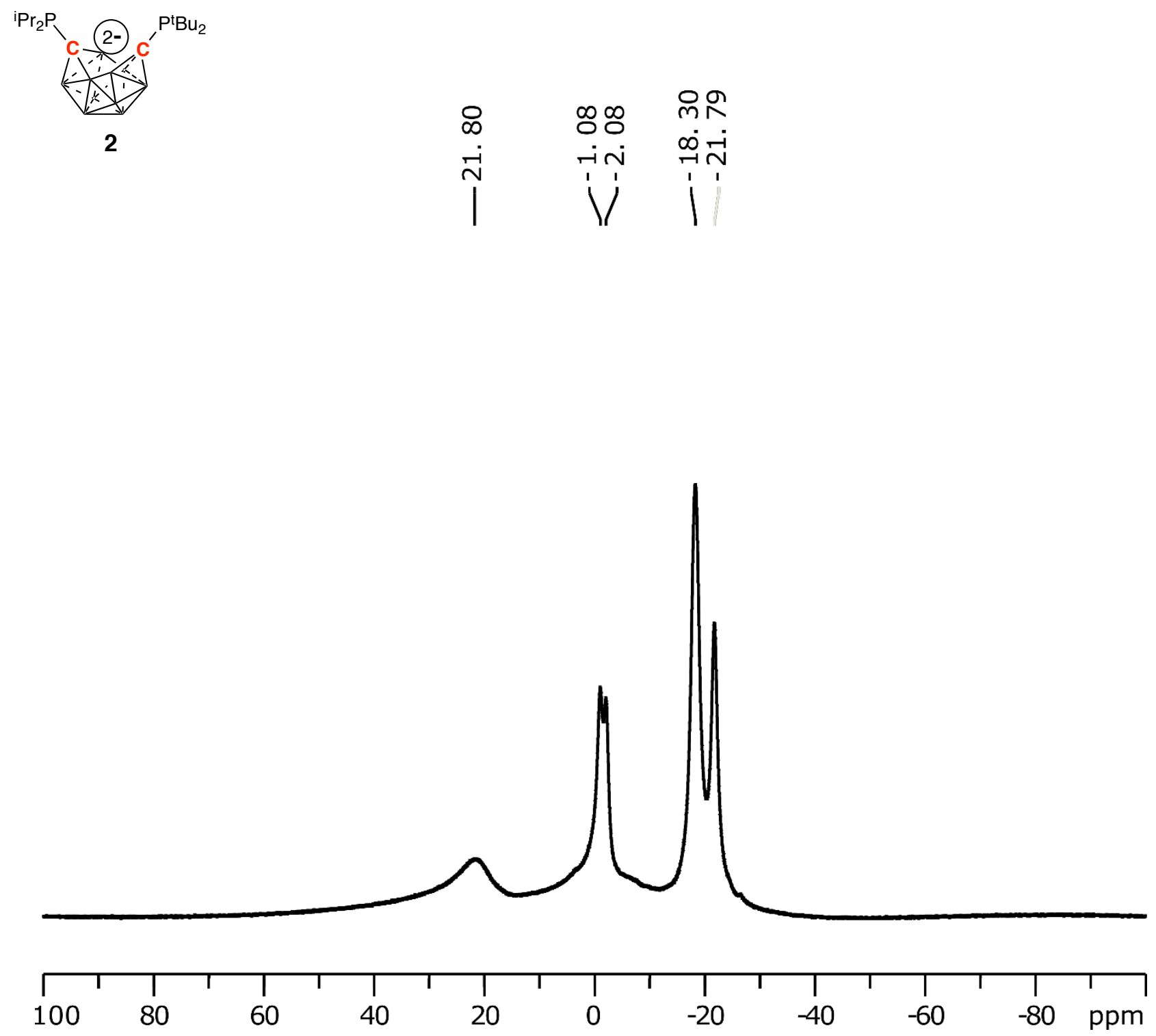

Figure S-6: The ${ }^{11} \mathrm{~B}\left\{{ }^{1} \mathrm{H}\right\}$ NMR spectrum of $\mathrm{Na}_{2}\left[\mathrm{P}^{\mathrm{t}} \mathrm{Bu}_{2}-\mathrm{Pi}^{\mathrm{P}} \mathrm{Pr}_{2}-\left(\mathrm{C}_{2} \mathrm{~B}_{10} \mathrm{H}_{10}\right)\right]$ (2).

${ }^{11} \mathbf{B}\left\{{ }^{1} \mathbf{H}\right\}$ NMR $\left(\right.$ THF-d $\left.\mathrm{d}_{8}\right): \delta 21.8(B \mathrm{H}),-1.1(B \mathrm{H}),-2.1(B \mathrm{H}),-18.3(B \mathrm{H}),-21.8(B \mathrm{H})$. 

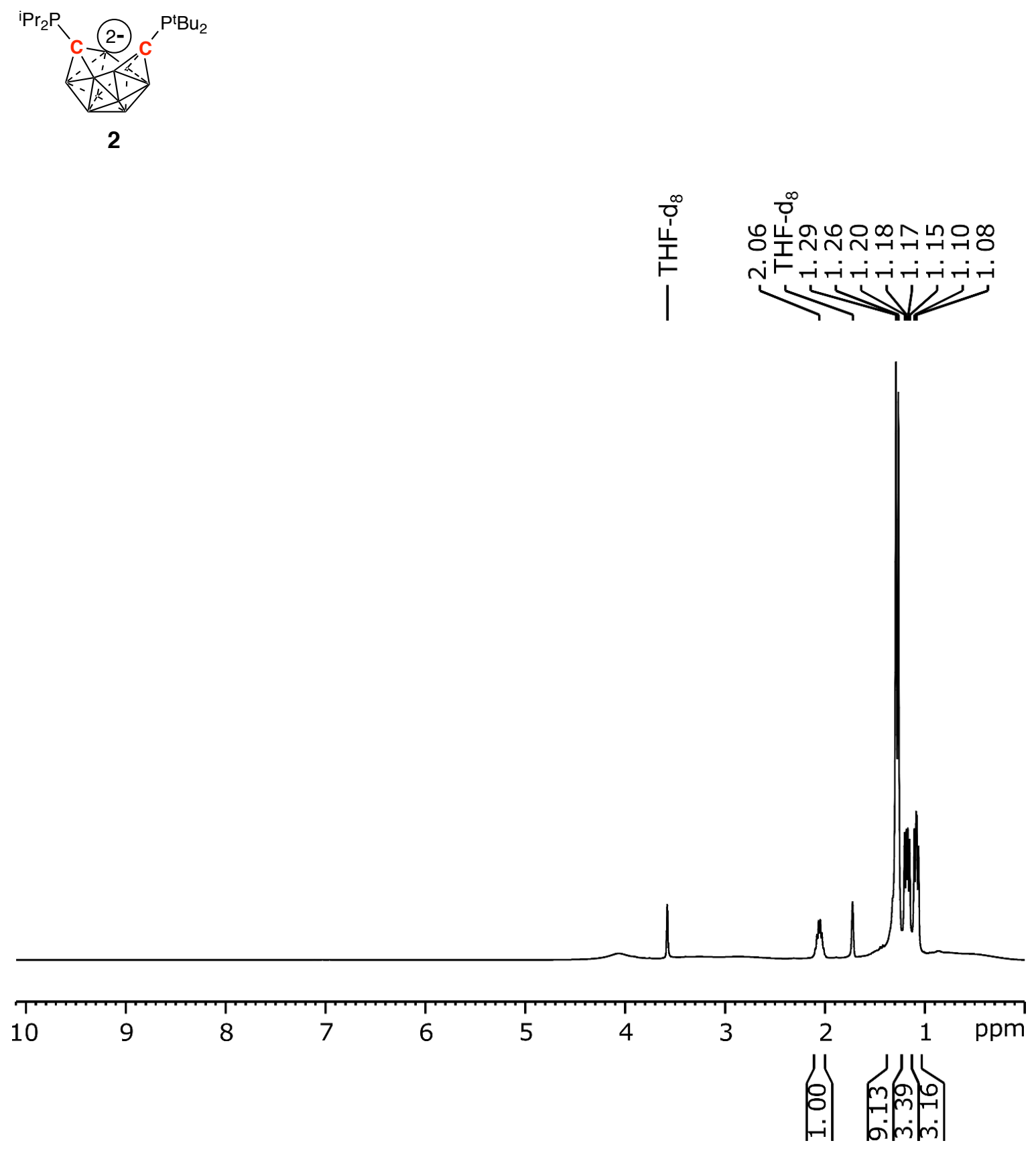

Figure S-7: The ${ }^{1} \mathrm{H}$ NMR spectrum of $\mathrm{Na}_{2}\left[\mathrm{P}^{t} \mathrm{Bu}_{2}-\mathrm{P}^{\mathrm{i}} \mathrm{Pr}_{2}-\left(\mathrm{C}_{2} \mathrm{~B}_{10} \mathrm{H}_{10}\right)\right](\mathbf{2})$.

${ }^{1}$ H NMR $\left(\mathrm{THF}_{-} \mathrm{d}_{8}\right): \delta 2.06\left(\mathrm{sp}, 2 \mathrm{H}, \mathrm{P}\left(\mathrm{CH}\left(\mathrm{CH}_{3}\right)_{2}\right)_{2}\right), 1.27\left(\mathrm{~d},{ }^{3} \mathrm{~J}_{\mathrm{P}-\mathrm{H}}=10 \mathrm{~Hz}, 18 \mathrm{H}, \mathrm{P}\left(\mathrm{C}\left(\mathrm{CH}_{3}\right)_{3}\right)_{2}\right)$, 1.20 to $1.08\left(\mathrm{~m}, 12 \mathrm{H}, \mathrm{P}\left(\mathrm{CH}\left(\mathrm{CH}_{3}\right)_{2}\right)_{2}\right)$. 

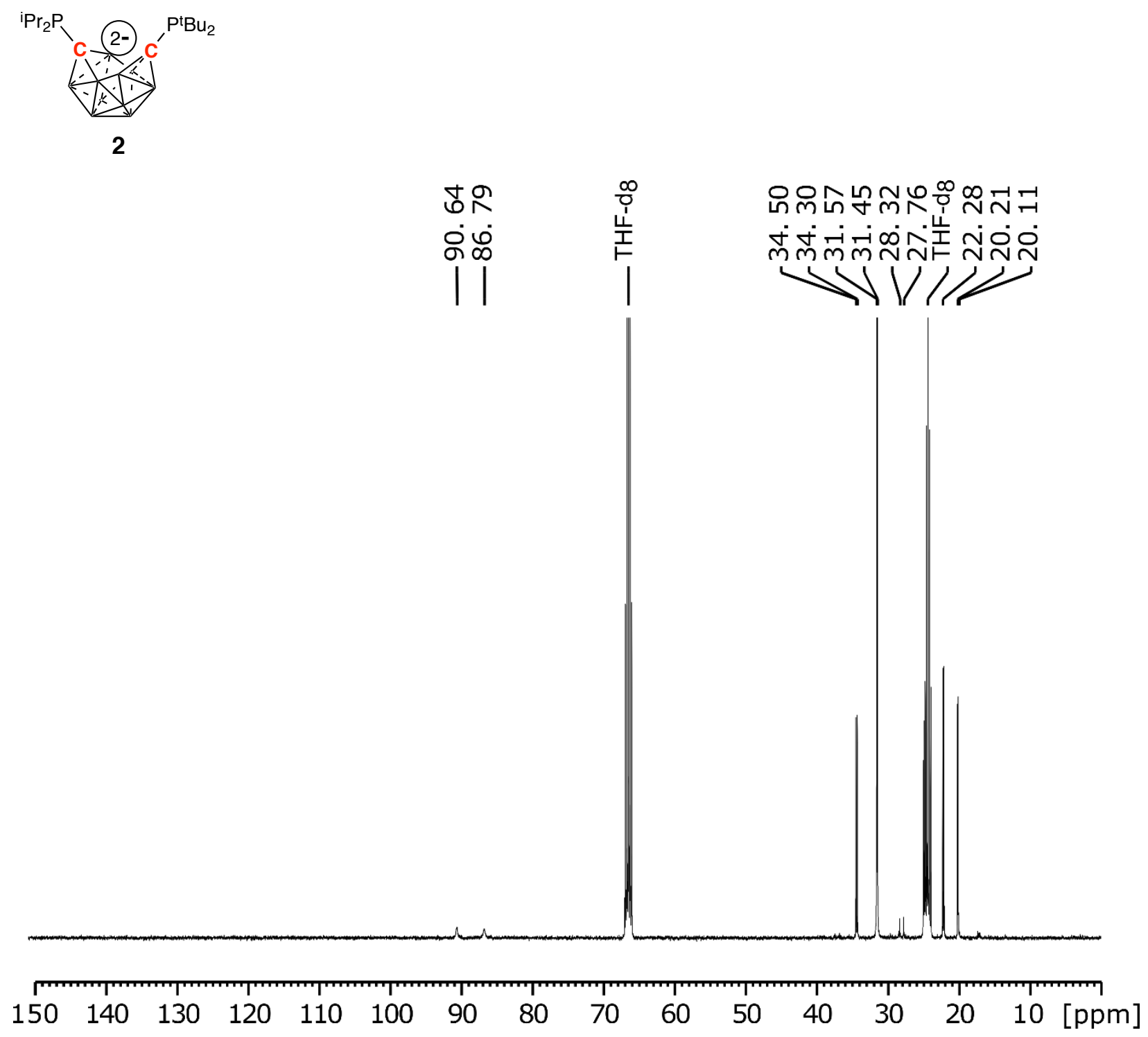

Figure S-8: The ${ }^{13} \mathrm{C}$ NMR spectrum of $\mathrm{Na}_{2}\left[\mathrm{PBu}_{2}-\mathrm{P}^{\mathrm{i}} \mathrm{Pr}_{2}-\left(\mathrm{C}_{2} \mathrm{~B}_{10} \mathrm{H}_{10}\right)\right]$ (2).

${ }^{13}$ C NMR (THF-d 8 ): 90.6 (s, CP (ortho-carborane)), 86.8 (s, CP (ortho-carborane)), 34.4 (d, ${ }^{1} J_{\mathrm{P}-}$ $\left.\mathrm{C}^{2}=20 \mathrm{~Hz}, \mathrm{P}\left(\mathrm{C}\left(\mathrm{CH}_{3}\right)_{3}\right)_{2}\right), 31.5\left(\mathrm{~d},{ }^{2} J_{\mathrm{P}-\mathrm{C}}=12 \mathrm{~Hz}, \mathrm{P}\left(\mathrm{C}\left(\mathrm{CH}_{3}\right)_{3}\right)_{2}\right), 28.0\left(\mathrm{~d},{ }^{1} J_{\mathrm{P}-\mathrm{C}}=56 \mathrm{~Hz}, \mathrm{P}\left(\mathrm{CH}\left(\mathrm{CH}_{3}\right)_{2}\right)_{2}\right)$, $22.2\left(\mathrm{~d},{ }^{2} J_{\mathrm{P}-\mathrm{C}}=18 \mathrm{~Hz}, \mathrm{P}\left(\mathrm{CH}\left(\mathrm{CH}_{3}\right)_{2}\right)_{2}\right), 20.2\left(\mathrm{~d},{ }^{2} J_{\mathrm{P}-\mathrm{C}}=10 \mathrm{~Hz}, \mathrm{P}\left(\mathrm{CH}\left(\mathrm{CH}_{3}\right)_{2}\right)_{2}\right)$. 

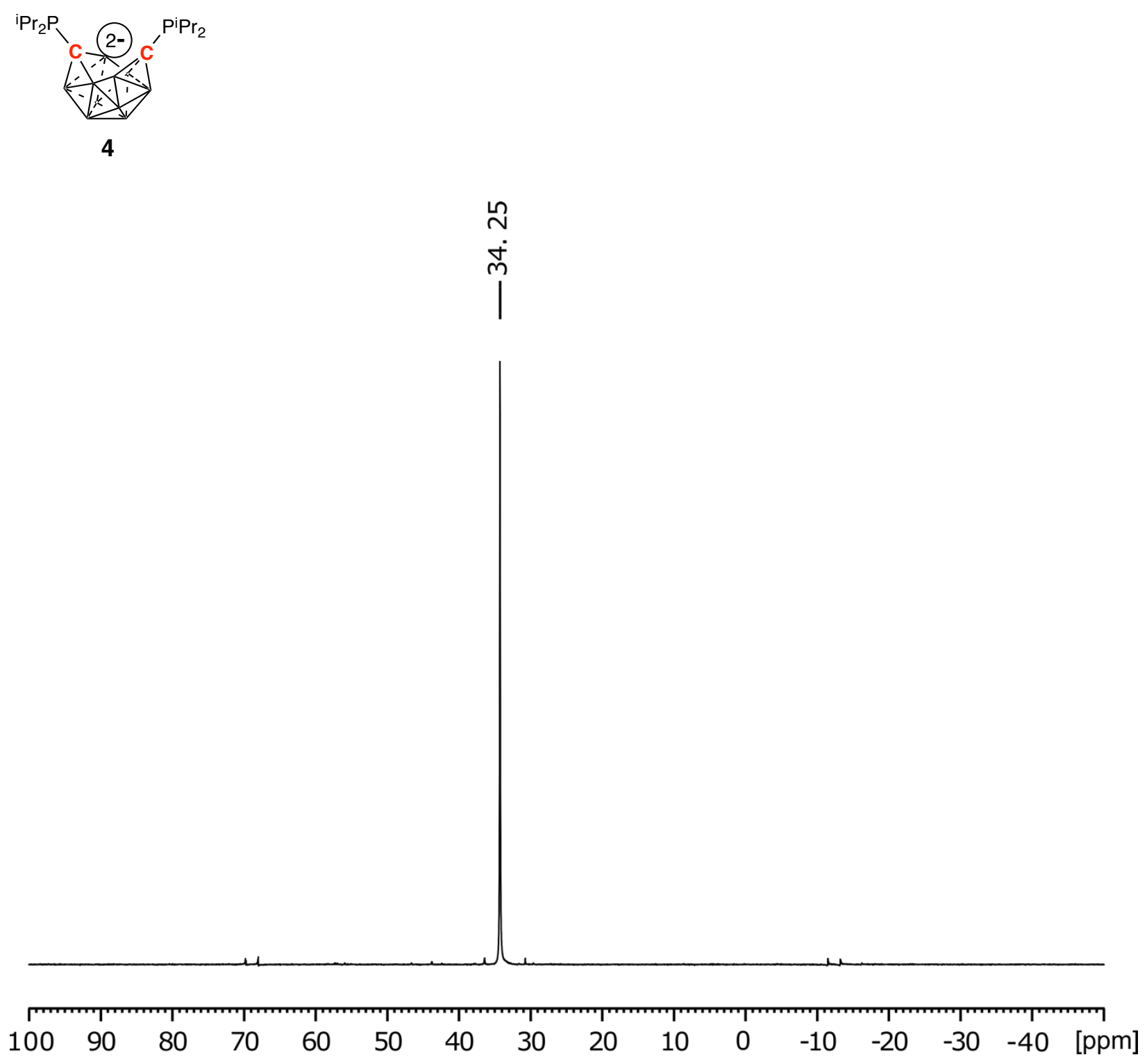

Figure S-9: The ${ }^{31} \mathrm{P}\left\{{ }^{1} \mathrm{H}\right\}$ NMR spectrum of $\mathrm{Na}_{2}\left[\left(\mathrm{P}^{\mathrm{i}} \mathrm{Pr}_{2}\right)_{2}\left(\mathrm{C}_{2} \mathrm{~B}_{10} \mathrm{H}_{10}\right)\right]$ (4).

${ }^{31} \mathbf{P}\left\{{ }^{1} \mathbf{H}\right\}$ NMR (THF-d 8$): \delta 34.3\left(\mathrm{~s}, P\left(\mathrm{CH}\left(\mathrm{CH}_{3}\right)_{2}\right)_{2}\right)$. 

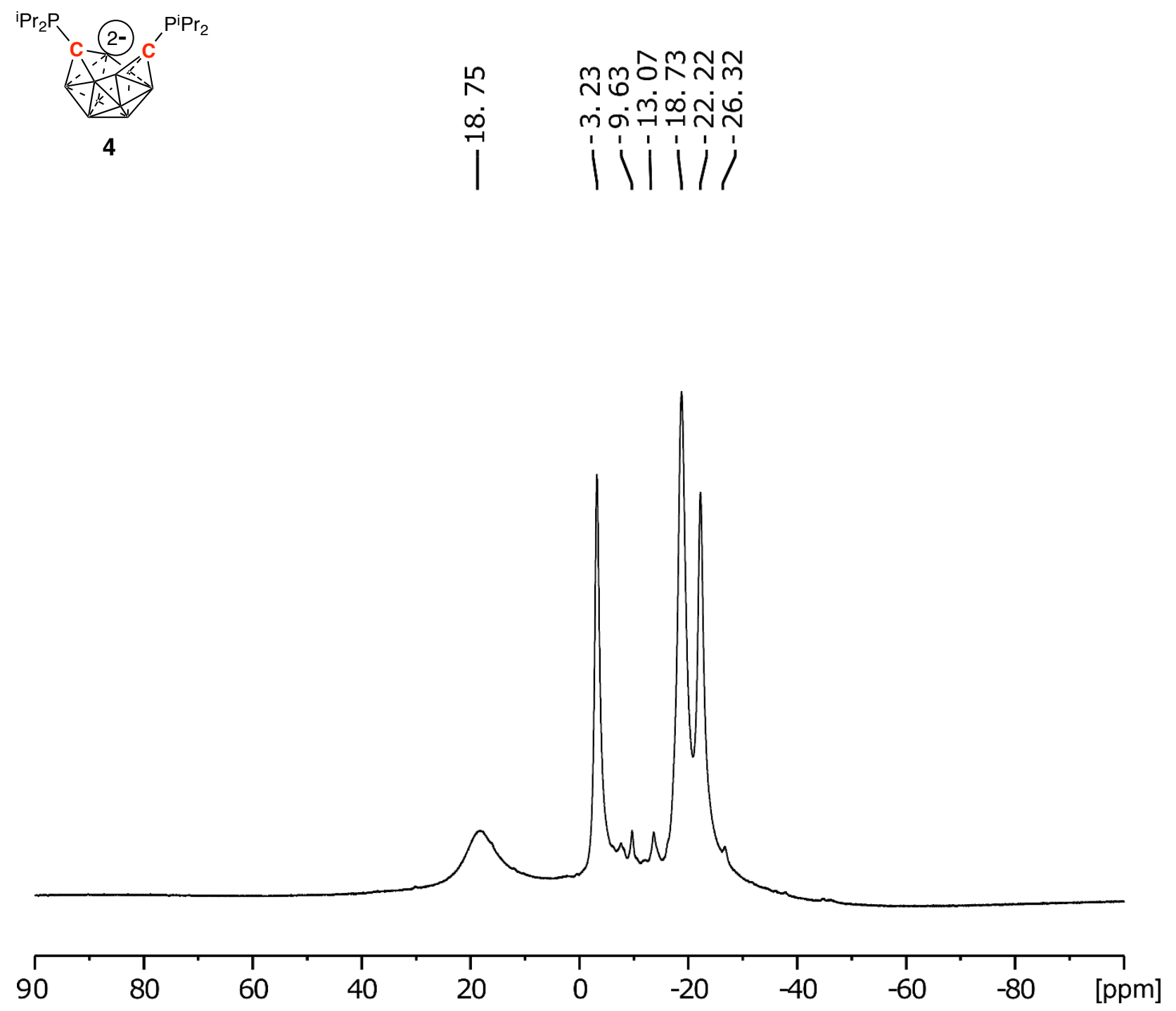

Figure S-10: The ${ }^{11} \mathrm{~B}\left\{{ }^{1} \mathrm{H}\right\}$ NMR spectrum of $\mathrm{Na}_{2}\left[\left(\mathrm{P}^{\mathrm{i}} \mathrm{Pr}_{2}\right)_{2}\left(\mathrm{C}_{2} \mathrm{~B}_{10} \mathrm{H}_{10}\right)\right](4)$.

${ }^{11} \mathbf{B}\left\{{ }^{1} \mathbf{H}\right\}$ NMR $\left(\right.$ THF-d $\left._{8}\right): \delta 18.8(B H),-3.2(B H),-9.6(B H),-13.1(B H),-18.7(B H),-22.2(B H),-$ $26.3(B \mathrm{H})$. 

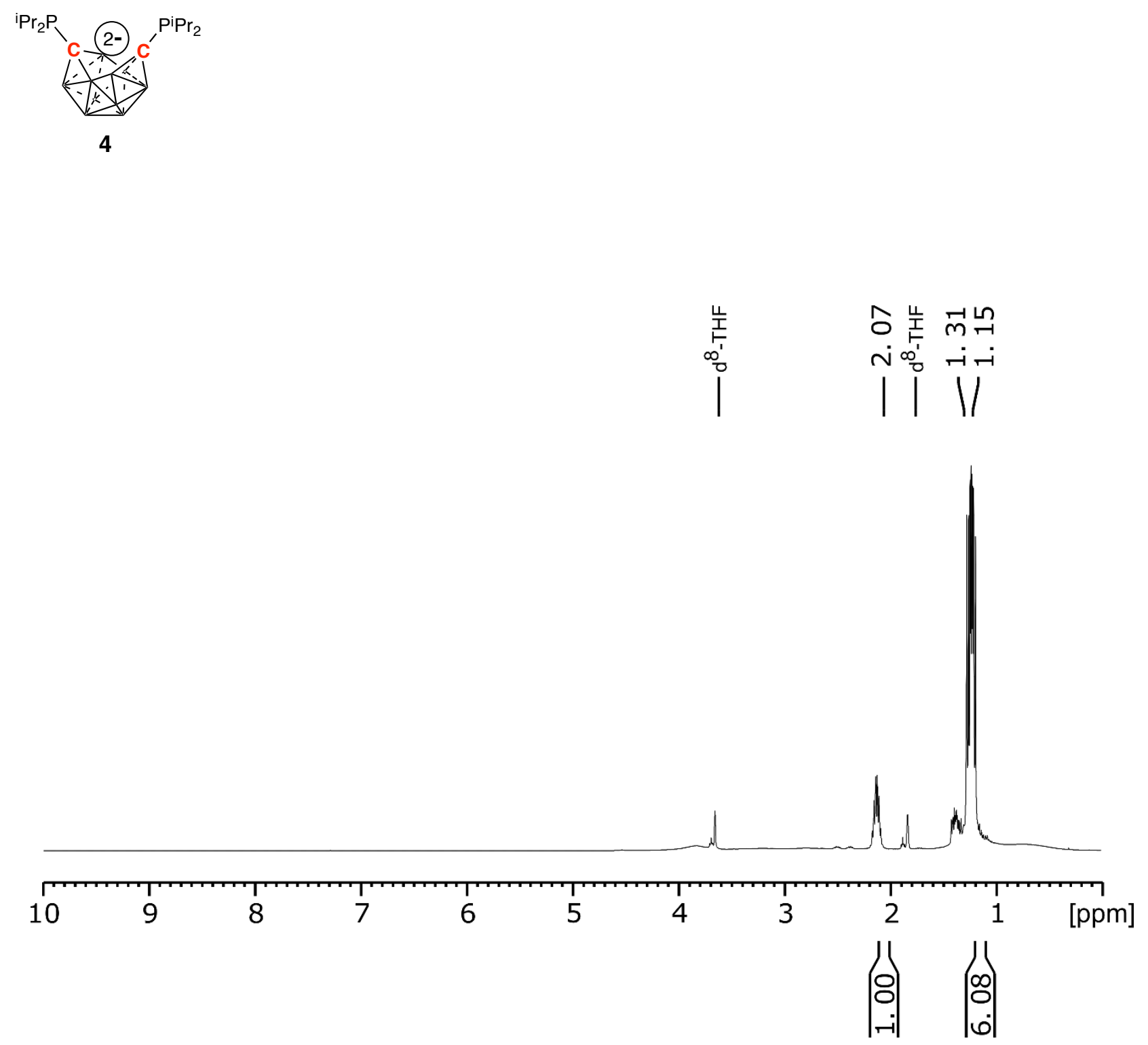

Figure S-11: The ${ }^{1} \mathrm{H}$ NMR spectrum of $\mathrm{Na}_{2}\left[\left(\mathrm{P}^{\mathrm{i}} \mathrm{Pr}_{2}\right)_{2}\left(\mathrm{C}_{2} \mathrm{~B}_{10} \mathrm{H}_{10}\right)\right]$ (4).

${ }^{1} \mathbf{H}$ NMR (THF-d $): \delta 2.07\left(\mathrm{sp}, 4 \mathrm{H}, \mathrm{P}\left(\mathrm{CH}\left(\mathrm{CH}_{3}\right)_{2}\right)_{2}\right), 1.15\left(\mathrm{~m}, 24 \mathrm{H}, \mathrm{P}\left(\mathrm{CH}\left(\mathrm{CH}_{3}\right)_{2}\right)_{2}\right)$. 


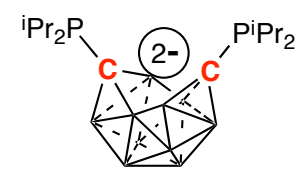

4

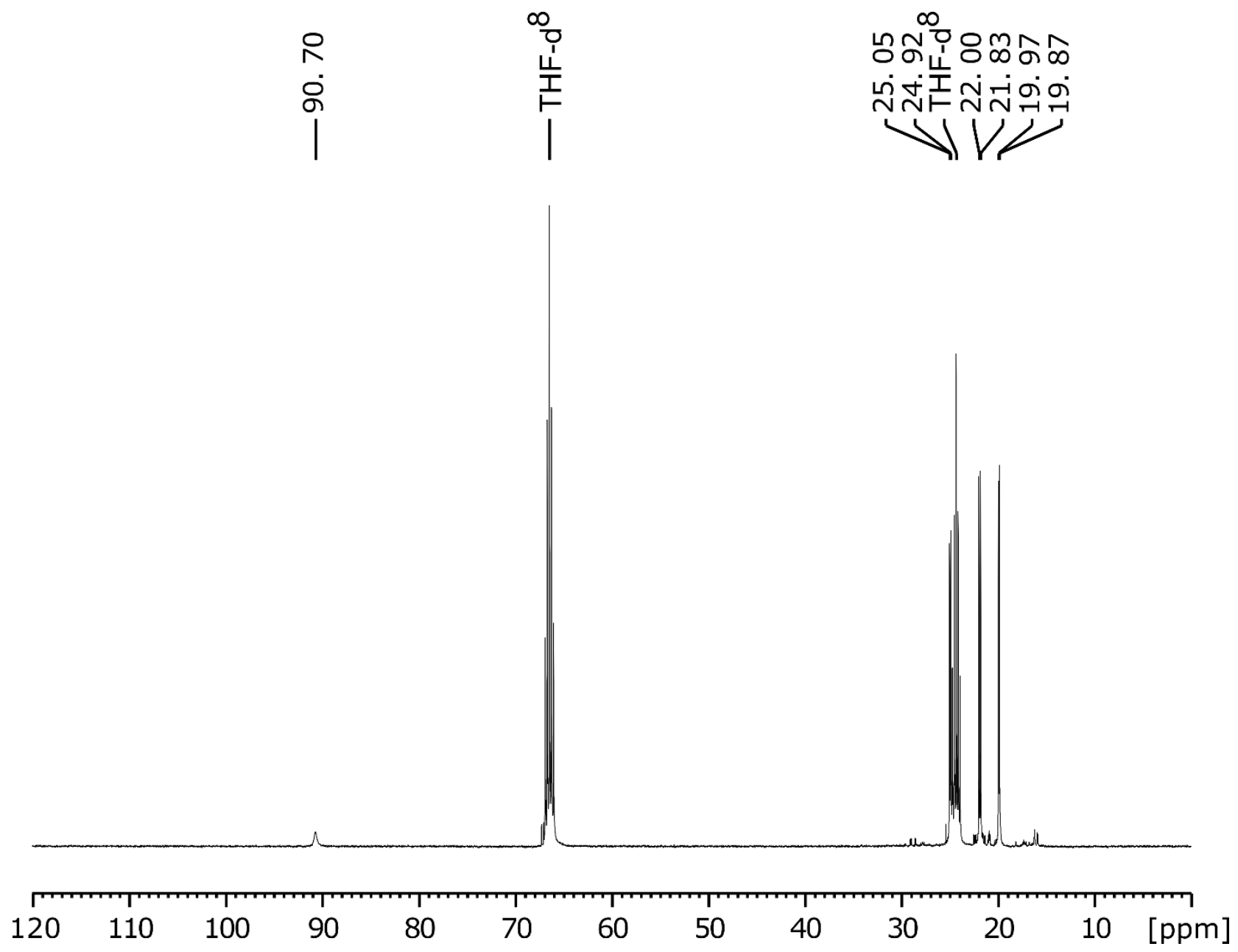

Figure S-12: The ${ }^{13} \mathrm{C}$ NMR spectrum of $\mathrm{Na}_{2}\left[\left(\mathrm{P}^{\mathrm{i}} \mathrm{Pr}_{2}\right)_{2}\left(\mathrm{C}_{2} \mathrm{~B}_{10} \mathrm{H}_{10}\right)\right]$ (4).

${ }^{13}$ C NMR (THF-d 8 ): 90.6 (s, CP (ortho-carborane)), 86.8 (s, CP (ortho-carborane)), 34.4 (d, ${ }^{1} J_{\mathrm{P}-\mathrm{C}}$ $\left.=20 \mathrm{~Hz}, \mathrm{P}\left(\mathrm{C}\left(\mathrm{CH}_{3}\right)_{3}\right)_{2}\right), 31.5\left(\mathrm{~d},{ }^{2} J_{\mathrm{P}-\mathrm{C}}=12 \mathrm{~Hz}, \mathrm{P}\left(\mathrm{C}\left(\mathrm{CH}_{3}\right)_{3}\right)_{2}\right), 28.0\left(\mathrm{~d},{ }^{1} J_{\mathrm{P}-\mathrm{C}}=56 \mathrm{~Hz}, \mathrm{P}\left(\mathrm{CH}\left(\mathrm{CH}_{3}\right)_{2}\right)_{2}\right)$, $22.2\left(\mathrm{~d},{ }^{2} J_{\mathrm{P}-\mathrm{C}}=18 \mathrm{~Hz}, \mathrm{P}\left(\mathrm{CH}\left(\mathrm{CH}_{3}\right)_{2}\right)_{2}\right), 20.2\left(\mathrm{~d},{ }^{2} J_{\mathrm{P}-\mathrm{C}}=10 \mathrm{~Hz}, \mathrm{P}\left(\mathrm{CH}\left(\mathrm{CH}_{3}\right)_{2}\right)_{2}\right)$. 

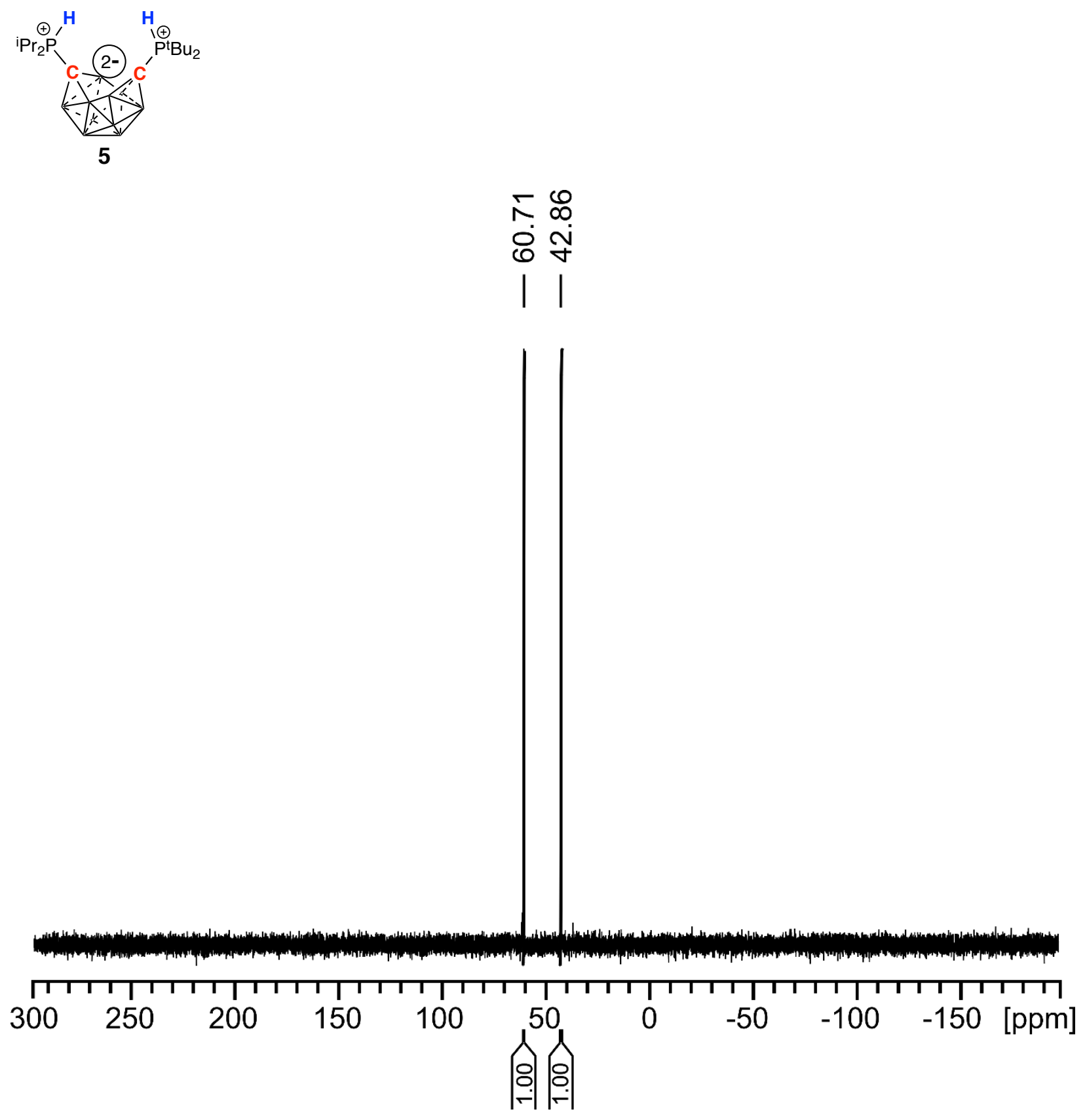

Figure S-13: The ${ }^{31} \mathrm{P}\left\{{ }^{1} \mathrm{H}\right\}$ NMR spectrum of $\mathrm{P}(\mathrm{H}){ }^{\mathrm{t}} \mathrm{Bu}_{2}-\mathrm{P}(\mathrm{H}){ }^{\mathrm{i}} \mathrm{Pr}_{2}-\left(\mathrm{C}_{2} \mathrm{~B}_{10} \mathrm{H}_{10}\right)(5)$.

${ }^{31} \mathbf{P}\left\{{ }^{1} \mathrm{H}\right\}$ NMR $\left(\mathrm{CD}_{2} \mathrm{Cl}_{2}\right): \delta 60.7\left(\mathrm{~s}, 1 \mathrm{P}, P\left(\mathrm{C}\left(\mathrm{CH}_{3}\right)_{3}\right)_{2}\right), 42.9\left(\mathrm{~s}, 1 \mathrm{P}, P\left(\mathrm{CH}\left(\mathrm{CH}_{3}\right)_{2}\right)_{2}\right)$. 

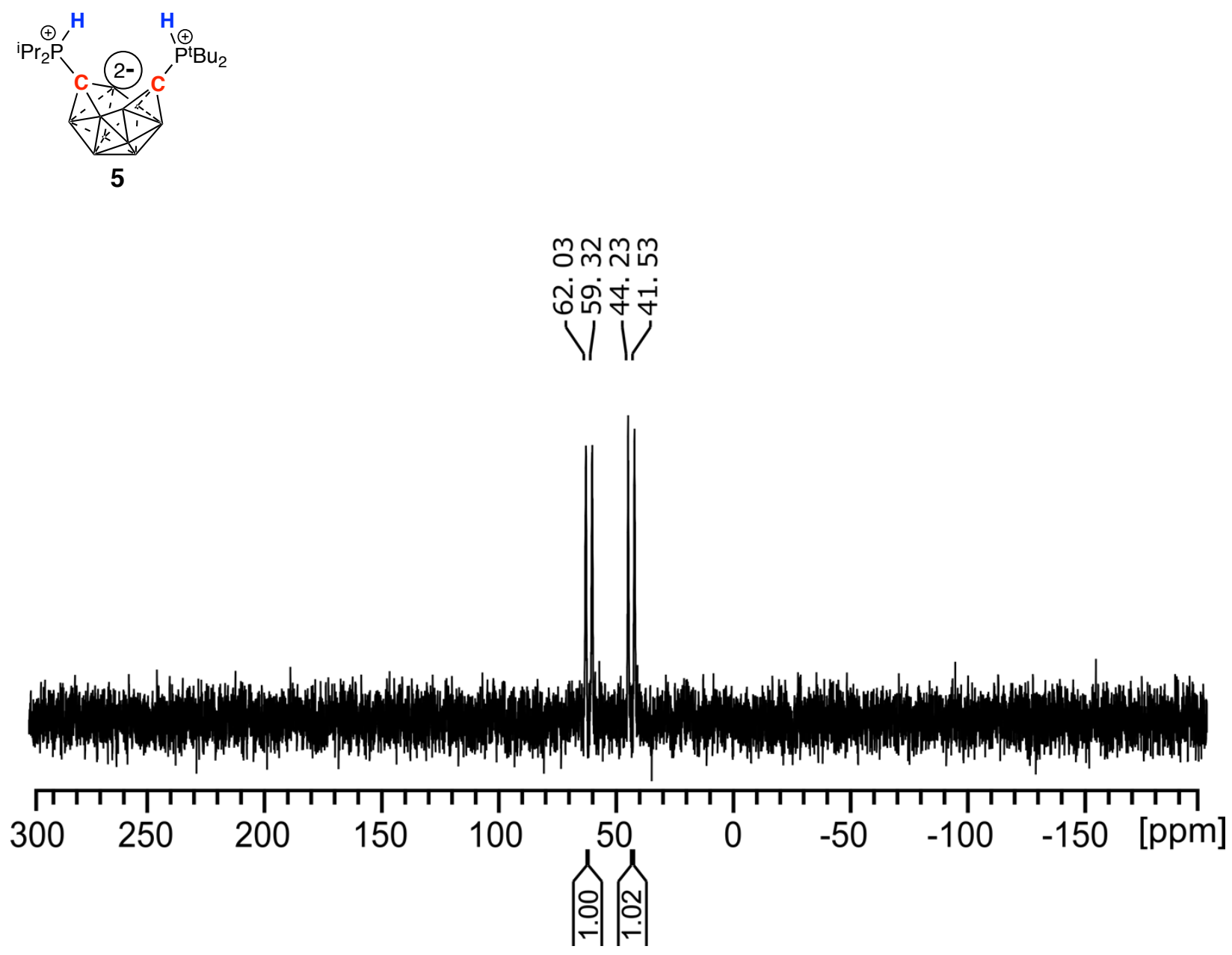

Figure S-14: The ${ }^{31} \mathrm{P}$ NMR spectrum of $\mathrm{P}(\mathrm{H})^{\mathrm{t}} \mathrm{Bu}_{2}-\mathrm{P}(\mathrm{H})^{\mathrm{i}} \mathrm{Pr}_{2}-\left(\mathrm{C}_{2} \mathrm{~B}_{10} \mathrm{H}_{10}\right)(5)$.

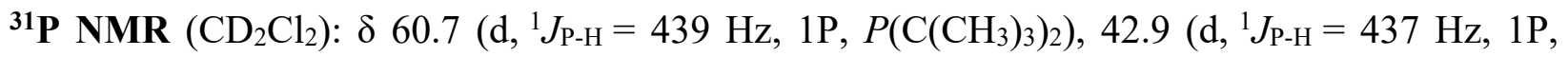
$\left.P\left(\mathrm{CH}\left(\mathrm{CH}_{3}\right)_{2}\right)_{2}\right)$. 

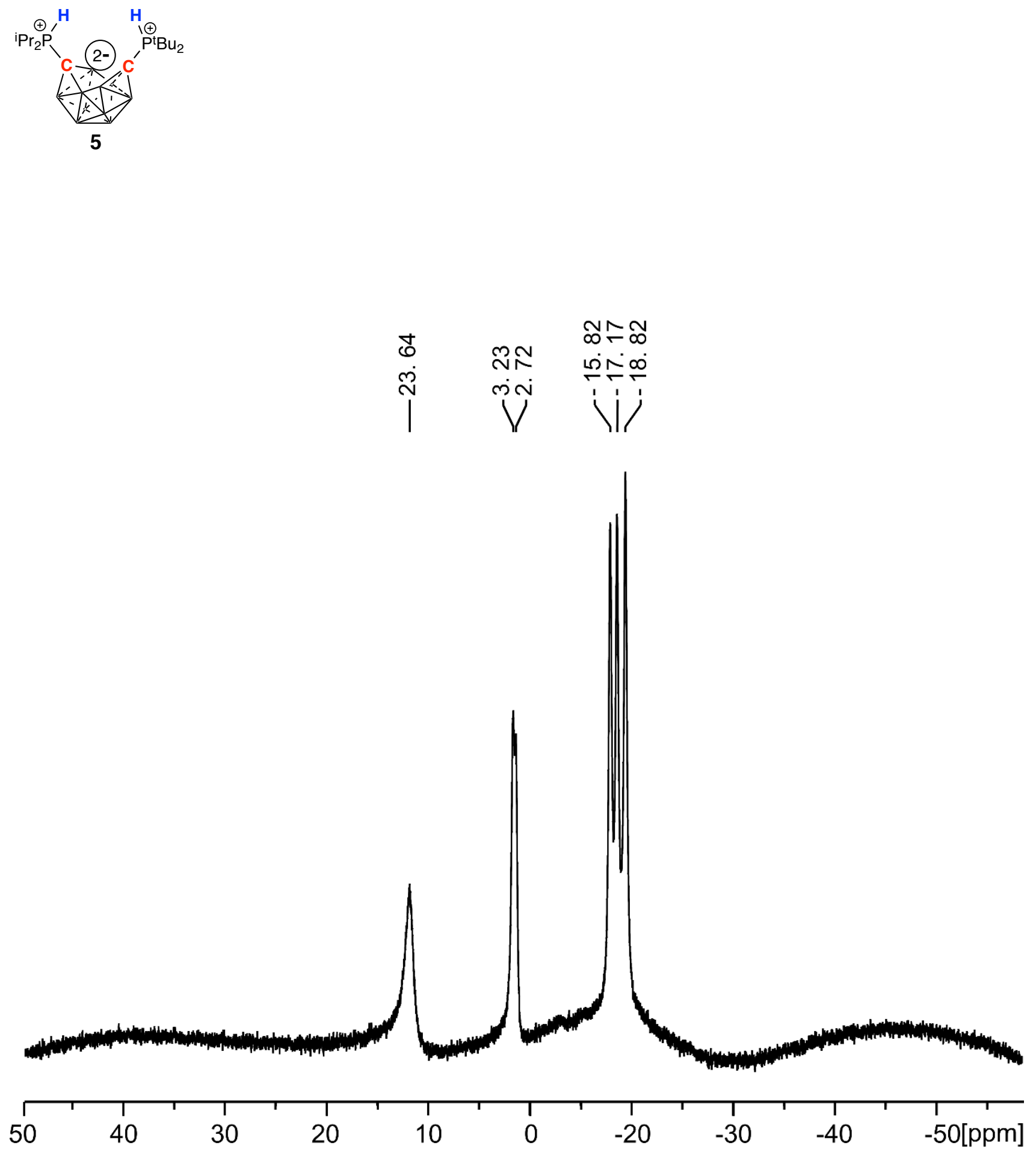

Figure S-15: The ${ }^{11} \mathrm{~B}\left\{{ }^{1} \mathrm{H}\right\}$ NMR spectrum of $\mathrm{P}(\mathrm{H})^{\mathrm{t}} \mathrm{Bu}_{2}-\mathrm{P}(\mathrm{H})^{\mathrm{i}} \mathrm{Pr}_{2}-\left(\mathrm{C}_{2} \mathrm{~B}_{10} \mathrm{H}_{10}\right)(5)$.

${ }^{11} \mathbf{B}\left\{{ }^{1} \mathrm{H}\right\}$ NMR $\left(\mathrm{CD}_{2} \mathrm{Cl}_{2}\right): \delta 23.6(B \mathrm{H}), 3.2(B \mathrm{H}), 2.7(B \mathrm{H}),-15.8(B \mathrm{H}),-17.2(B \mathrm{H}),-18.8(B \mathrm{H})$ 

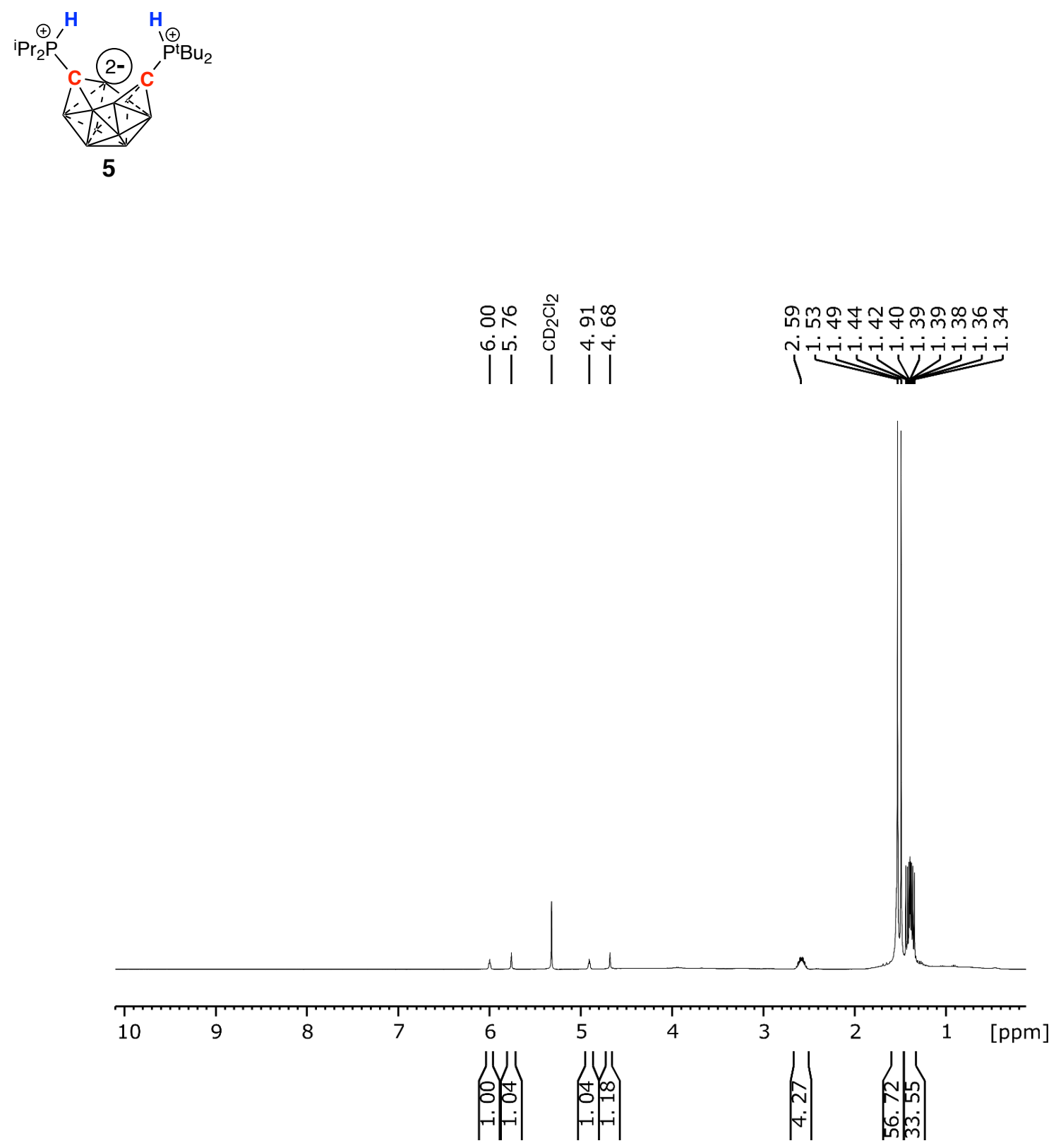

Figure S-16: The ${ }^{1} \mathrm{H}$ NMR spectrum of $\mathrm{P}(\mathrm{H})^{\mathrm{t}} \mathrm{Bu}_{2}-\mathrm{P}(\mathrm{H}){ }^{\mathrm{i}} \mathrm{Pr}_{2}-\left(\mathrm{C}_{2} \mathrm{~B}_{10} \mathrm{H}_{10}\right)(5)$.

${ }^{1} \mathbf{H}$ NMR $\left(\mathrm{CD}_{2} \mathrm{Cl}_{2}\right): \delta 5.46\left(\mathrm{td},{ }^{1} J_{\mathrm{P}-\mathrm{H}}=437 \mathrm{~Hz},{ }^{3} J_{\mathrm{H}-\mathrm{H}}=3 \mathrm{~Hz}, 1 \mathrm{H}, H-\mathrm{P}\left(\mathrm{CH}\left(\mathrm{CH}_{3}\right)_{2}\right)_{2}\right), 5.22\left(\mathrm{~d},{ }^{1} J_{\mathrm{P}-\mathrm{H}}\right.$ $\left.=433 \mathrm{~Hz}, 1 \mathrm{H}, \mathrm{H}-\mathrm{P}\left(\mathrm{C}\left(\mathrm{CH}_{3}\right)_{3}\right)_{2}\right), 2.59\left(\mathrm{~m}, 2 \mathrm{H}, \mathrm{P}\left(\mathrm{CH}\left(\mathrm{CH}_{3}\right)_{2}\right)_{2}\right), 1.51\left(\mathrm{~d},{ }^{3} J_{\mathrm{P}-\mathrm{H}}=15 \mathrm{~Hz}, 18 \mathrm{H}\right.$, $\left.\mathrm{P}\left(\mathrm{C}\left(\mathrm{CH}_{3}\right)_{3}\right)_{2}\right), 1.39\left(\mathrm{~m}, 12 \mathrm{H}, \mathrm{P}\left(\mathrm{CH}\left(\mathrm{CH}_{3}\right)_{2}\right)_{2}\right)$. 

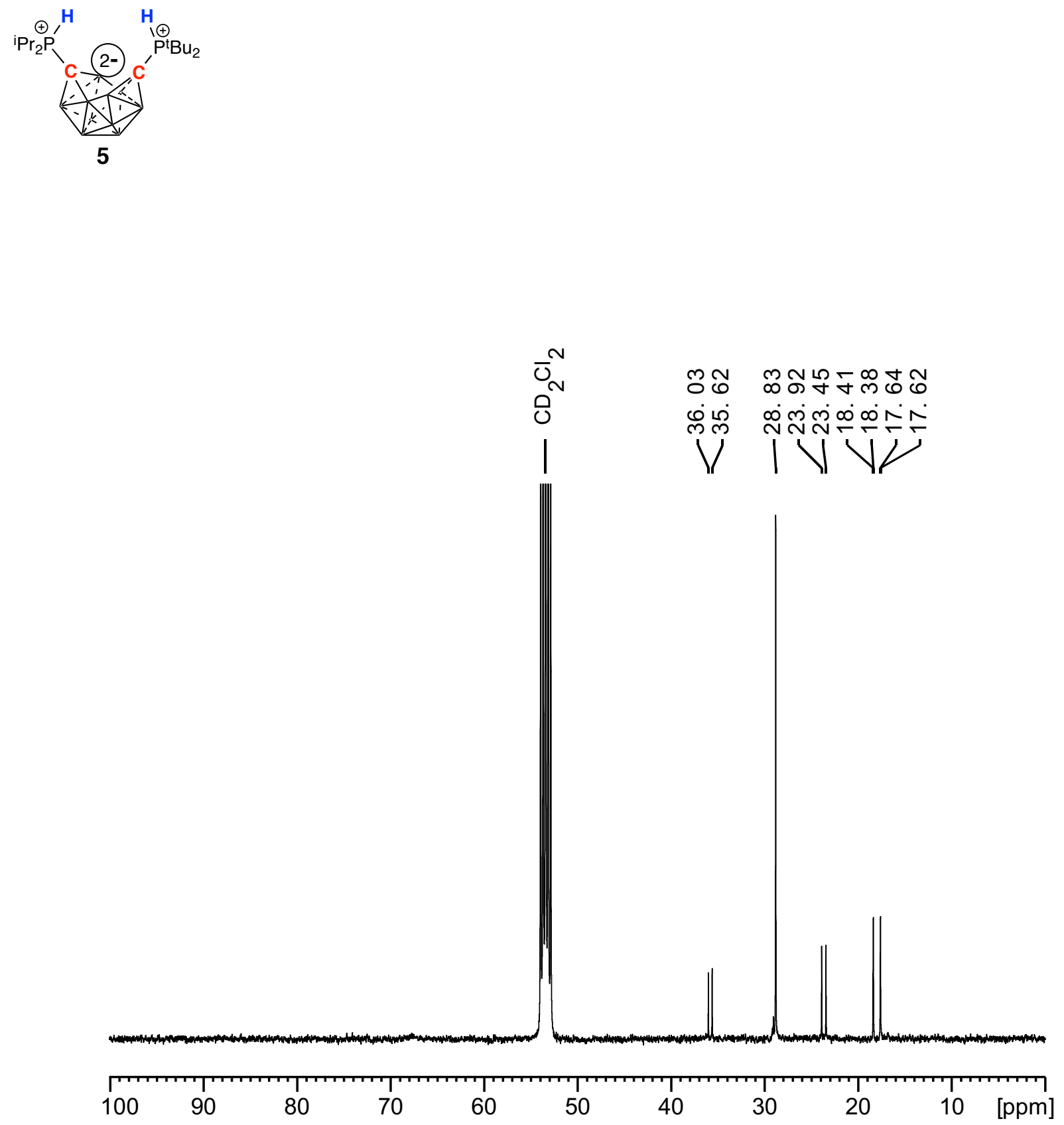

Figure S-17: The ${ }^{13} \mathrm{C}$ NMR spectrum of $\mathrm{P}(\mathrm{H})^{\mathrm{t}} \mathrm{Bu} 2-\mathrm{P}(\mathrm{H}){ }^{\mathrm{i}} \mathrm{Pr}_{2}-\left(\mathrm{C}_{2} \mathrm{~B}_{10} \mathrm{H}_{10}\right)(\mathbf{5})$.

${ }^{13} \mathrm{C}$ NMR $\left(\mathrm{CD}_{2} \mathrm{Cl}_{2}\right): \delta 35.8\left(\mathrm{~d},{ }^{1} J_{\mathrm{C}-\mathrm{P}}=41 \mathrm{~Hz}, \mathrm{P}\left(C\left(\mathrm{CH}_{3}\right)_{3}\right)_{2}\right), 28.8\left(\mathrm{~s}, \mathrm{P}\left(\mathrm{C}\left(\mathrm{CH}_{3}\right)_{3}\right)_{2}\right), 23.7\left(\mathrm{~d},{ }^{1} J_{\mathrm{C}-\mathrm{P}}=\right.$ $\left.47 \mathrm{~Hz}, \mathrm{P}\left(\mathrm{CH}\left(\mathrm{CH}_{3}\right)_{2}\right)_{2}\right), 18.4\left(\mathrm{~d},{ }^{2} J_{\mathrm{C}-\mathrm{P}}=2 \mathrm{~Hz}, \mathrm{P}\left(\mathrm{CH}\left(\mathrm{CH}_{3}\right)_{2}\right)_{2}\right), 17.6\left(\mathrm{~d},{ }^{2} \mathrm{~J}_{\mathrm{C}-\mathrm{P}}=2 \mathrm{~Hz}, \mathrm{P}\left(\mathrm{CH}\left(\mathrm{CH}_{3}\right)_{2}\right)_{2}\right)$. 

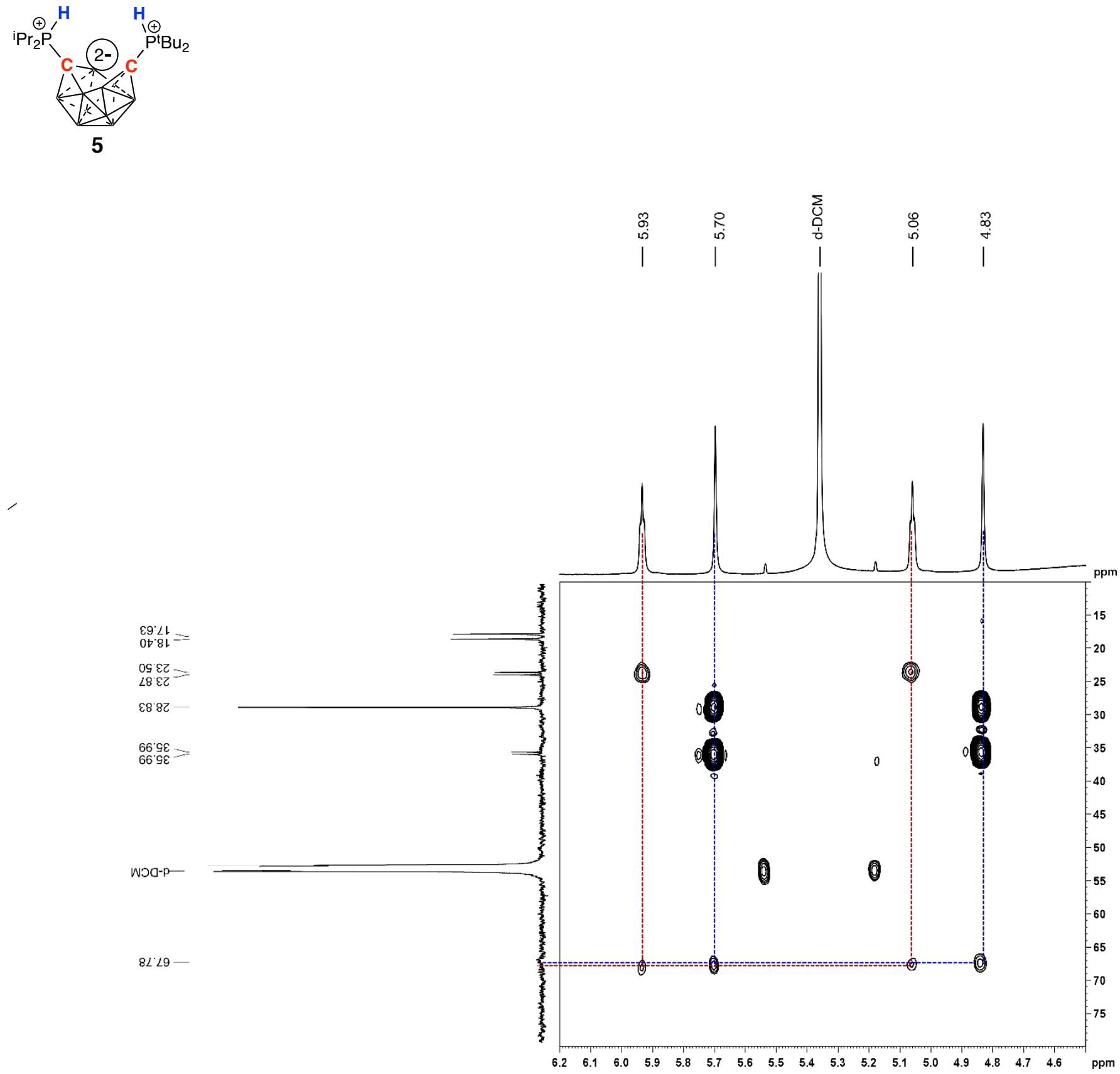

Figure S-18: The ${ }^{1} \mathrm{H}_{-}{ }^{13} \mathrm{C}$ HMBC NMR spectrum of $\mathrm{P}(\mathrm{H})^{\mathrm{t}} \mathrm{Bu}_{2}-\mathrm{P}(\mathrm{H})^{\mathrm{i}} \mathrm{Pr}_{2}-\left(\mathrm{C}_{2} \mathrm{~B}_{10} \mathrm{H}_{10}\right)$ (5). The spectrum demonstrates correlations between carbon atoms of the open carborane cluster and hydrogen atoms on the protonated phosphine centers. 

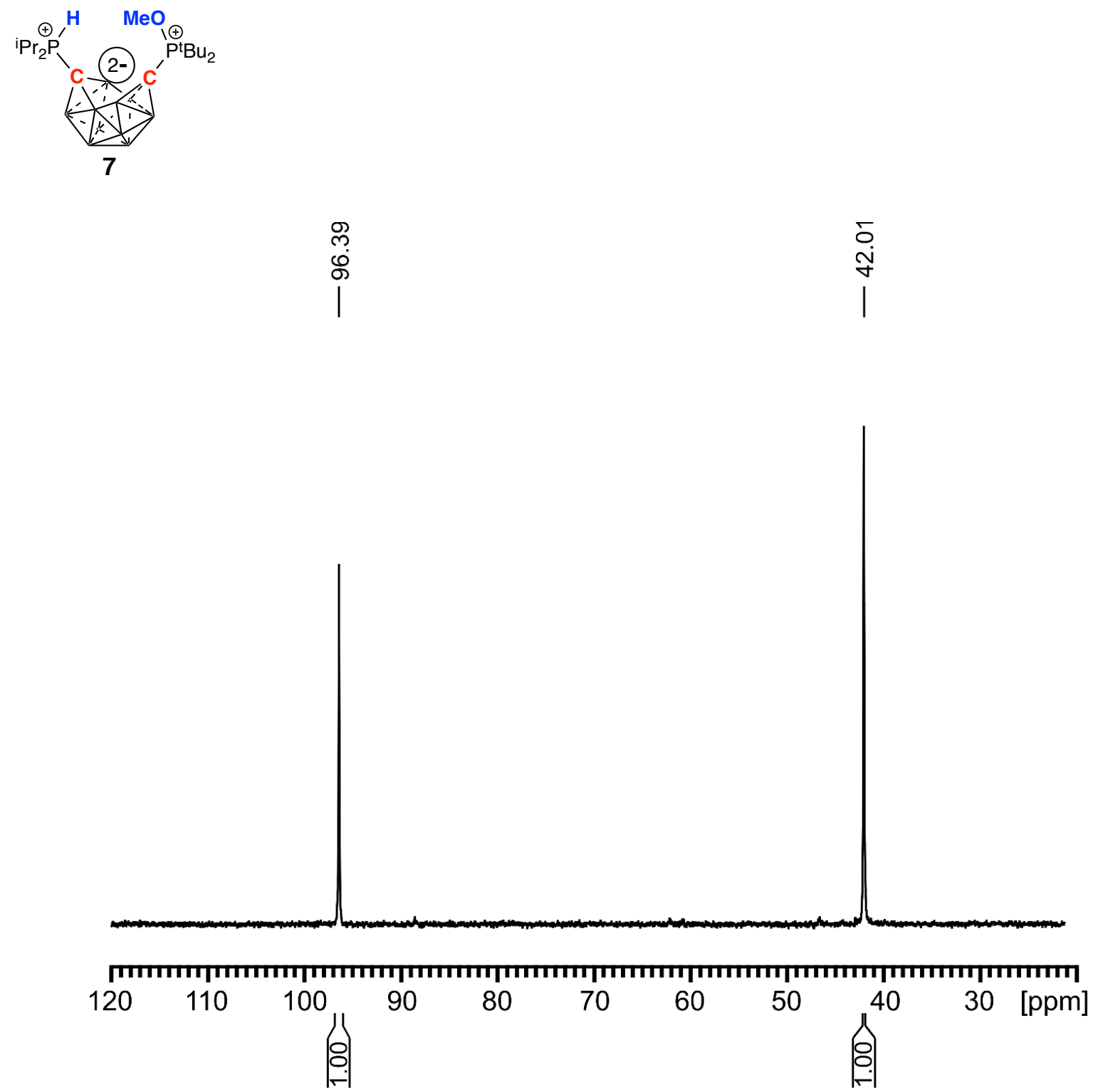

Figure S-19: The ${ }^{31} \mathrm{P}\left\{{ }^{1} \mathrm{H}\right\}$ NMR spectrum of $\mathrm{P}^{t} \mathrm{Bu}_{2}(\mathrm{OMe})-\mathrm{P}^{\mathrm{i}} \mathrm{Pr}_{2}(\mathrm{H})-\left(\mathrm{C}_{2} \mathrm{~B}_{10} \mathrm{H}_{10}\right)(7)$.

${ }^{31} \mathbf{P}\left\{{ }^{1} \mathrm{H}\right\}$ NMR $\left(\mathrm{CD}_{2} \mathrm{Cl}_{2}\right): \delta 96.4\left(\mathrm{~s}, 1 \mathrm{P}, P\left(\mathrm{C}\left(\mathrm{CH}_{3}\right)_{3}\right)_{2}\right), 42.0\left(\mathrm{~s}, 1 \mathrm{P}, P\left(\mathrm{CH}\left(\mathrm{CH}_{3}\right)_{2}\right)_{2}\right)$. 

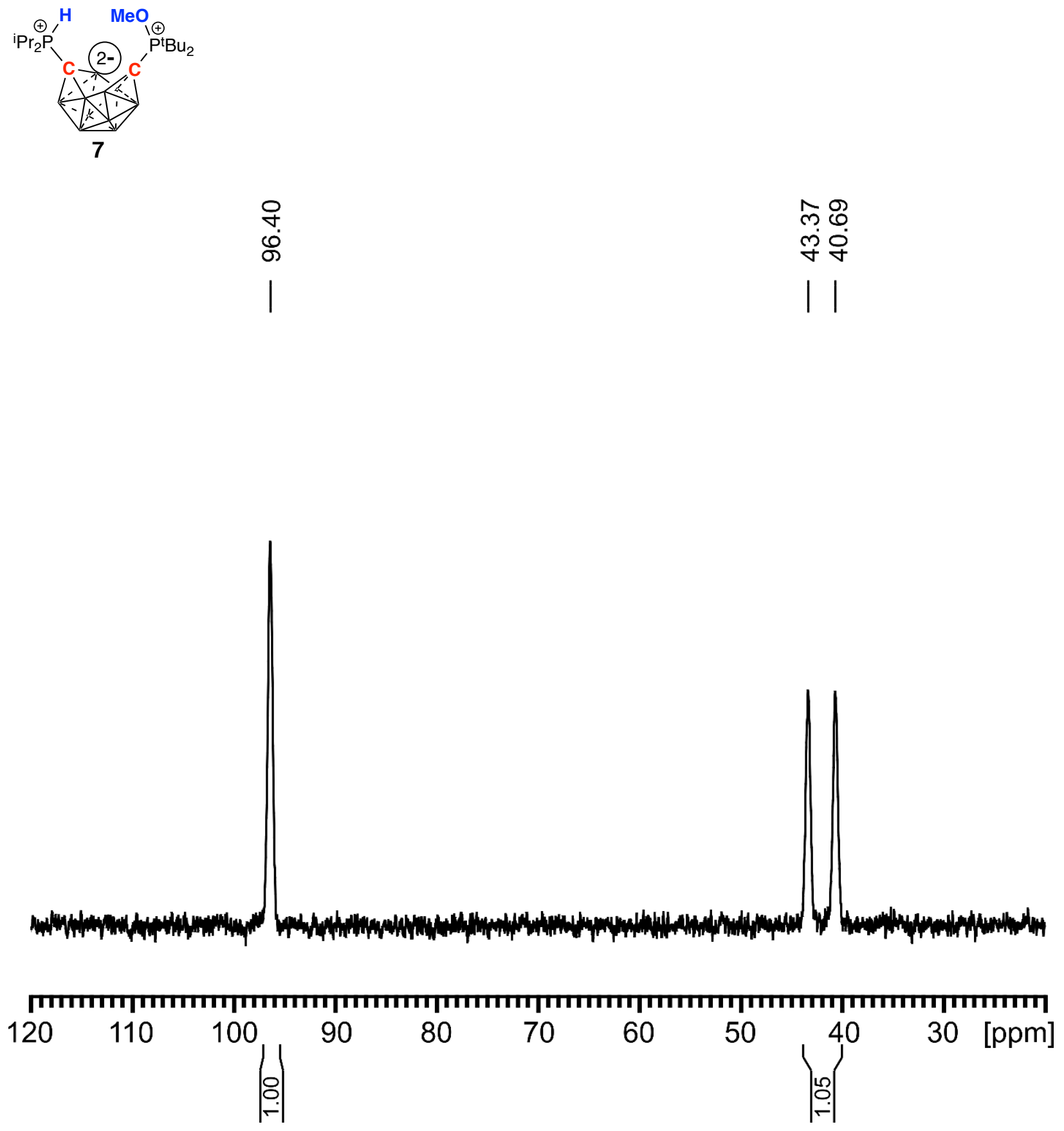

Figure S-20: The ${ }^{31} \mathrm{P}$ NMR spectrum of $\mathrm{P}^{\mathrm{t}} \mathrm{Bu}_{2}(\mathrm{OMe})-\mathrm{P}^{\mathrm{i}} \mathrm{Pr}_{2}(\mathrm{H})-\left(\mathrm{C}_{2} \mathrm{~B}_{10} \mathrm{H}_{10}\right)(7)$.

${ }^{31} \mathbf{P}$ NMR $\left(\mathrm{CD}_{2} \mathrm{Cl}_{2}\right): \delta 96.4\left(\mathrm{~s}, 1 \mathrm{P}, P\left(\mathrm{C}\left(\mathrm{CH}_{3}\right)_{3}\right)_{2}\right), 42.0\left(\mathrm{~d},{ }^{1} \mathrm{~J}_{\mathrm{P}-\mathrm{H}}=435 \mathrm{~Hz}, 1 \mathrm{P}, \mathrm{H}-P\left(\mathrm{CH}\left(\mathrm{CH}_{3}\right)_{2}\right)_{2}\right)$. 

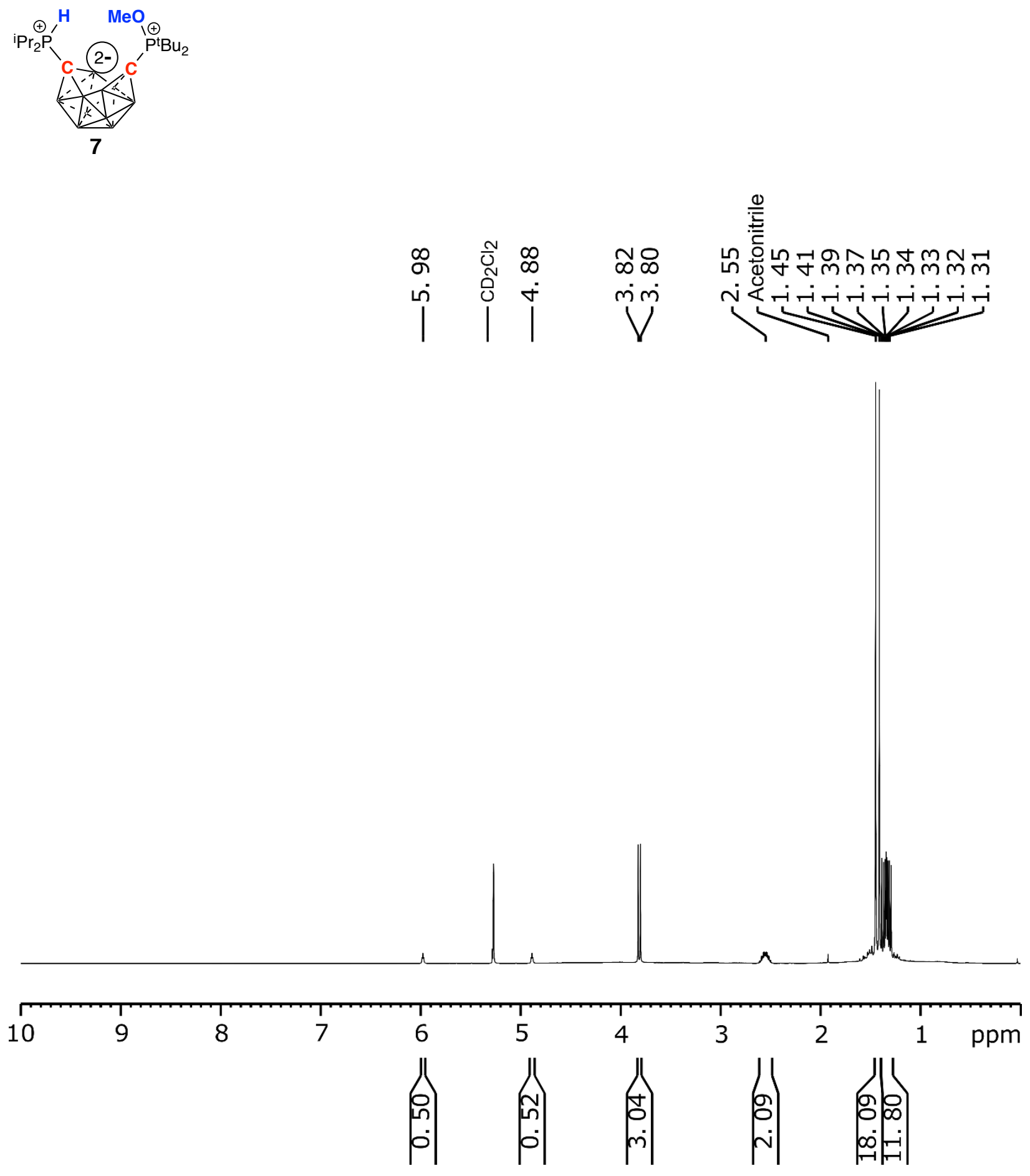

Figure S-21: The ${ }^{1} \mathrm{H}$ NMR spectrum of $\mathrm{P}^{\mathrm{t}} \mathrm{Bu}_{2}(\mathrm{OMe})-\mathrm{P}^{\mathrm{i}} \mathrm{Pr}_{2}(\mathrm{H})-\left(\mathrm{C}_{2} \mathrm{~B}_{10} \mathrm{H}_{10}\right)(7)$.

${ }^{1} \mathbf{H}$ NMR $\left(\mathrm{CD}_{2} \mathrm{Cl}_{2}\right): \delta 5.43\left(\mathrm{td},{ }^{1} J_{\mathrm{P}-\mathrm{H}}=438 \mathrm{~Hz},{ }^{3} J_{\mathrm{H}-\mathrm{H}}=3 \mathrm{~Hz}, 1 \mathrm{H}, H-\mathrm{P}\left(\mathrm{CH}\left(\mathrm{CH}_{3}\right)_{2}\right)_{2}\right), 3.81\left(\mathrm{~d},{ }^{3} J_{\mathrm{P}-\mathrm{H}}\right.$ $\left.=10 \mathrm{~Hz}, 3 \mathrm{H}, \mathrm{OCH}_{3}\right), 2.55\left(\mathrm{~m}, 2 \mathrm{H}, \mathrm{P}\left(\mathrm{CH}\left(\mathrm{CH}_{3}\right)_{2}\right)_{2}\right), 1.43\left(\mathrm{~d},{ }^{3} J_{\mathrm{P}-\mathrm{H}}=15 \mathrm{~Hz}, 18 \mathrm{H}, \mathrm{P}\left(\mathrm{C}\left(\mathrm{CH}_{3}\right)_{3}\right)_{2}\right), 1.34$ (m, 12H, $\left.\mathrm{P}\left(\mathrm{CH}\left(\mathrm{CH}_{3}\right)_{2}\right)_{2}\right)$. 

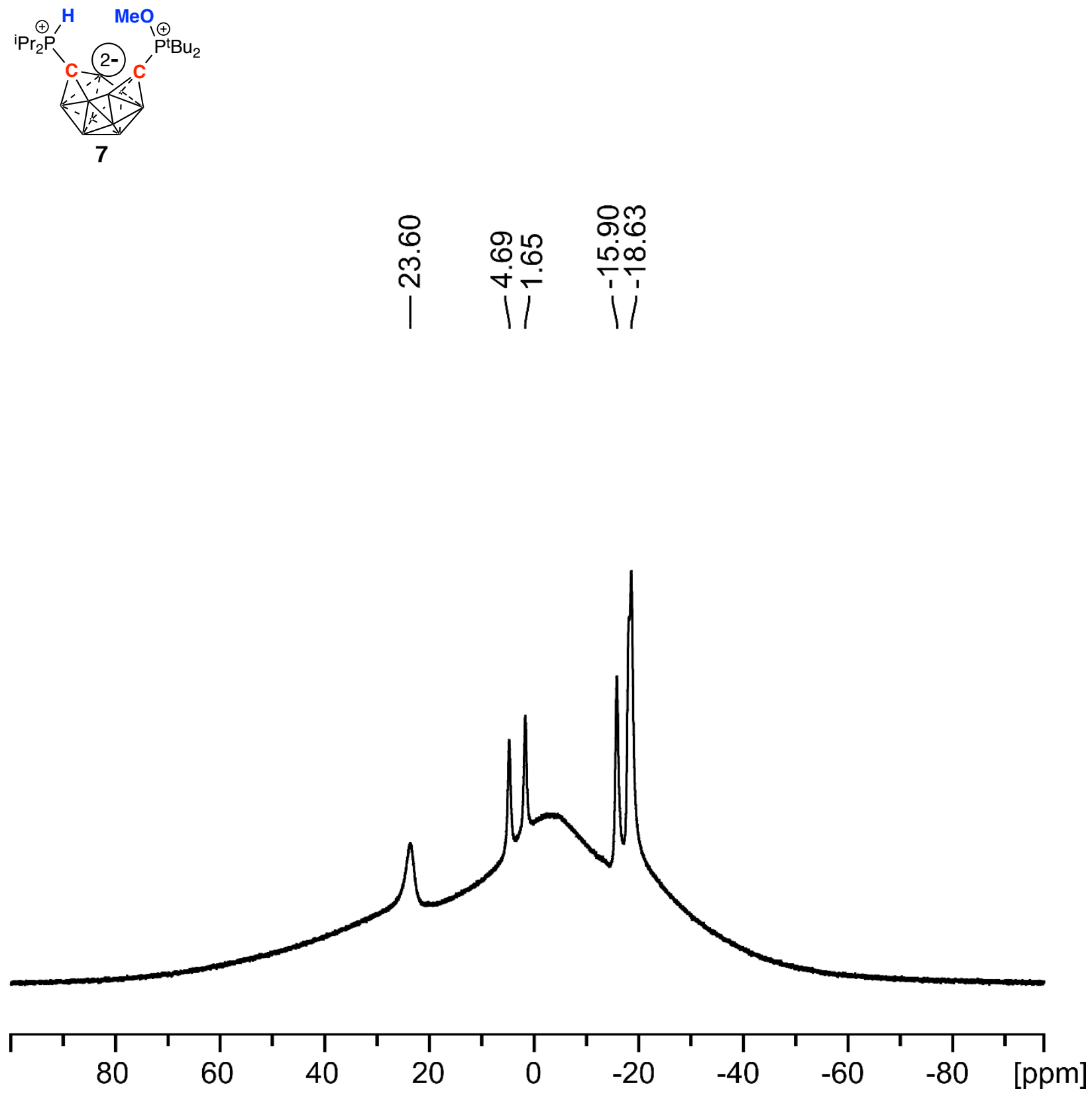

Figure S-22: The ${ }^{11} \mathrm{~B}\left\{{ }^{1} \mathrm{H}\right\}$ NMR spectrum of $\mathrm{P}^{\mathrm{t}} \mathrm{Bu}_{2}(\mathrm{OMe})-\mathrm{Pi}^{\mathrm{P}} \mathrm{Pr}_{2}(\mathrm{H})-\left(\mathrm{C}_{2} \mathrm{~B}_{10} \mathrm{H}_{10}\right)(7)$.

${ }^{11} \mathbf{B}\left\{{ }^{1} \mathbf{H}\right\}$ NMR $\left(\mathrm{CD}_{2} \mathrm{Cl}_{2}\right): \delta 23.6(B \mathrm{H}), 4.7(B \mathrm{H}), 1.7(B \mathrm{H}),-15.9(B \mathrm{H}),-18.6(B \mathrm{H})$. 

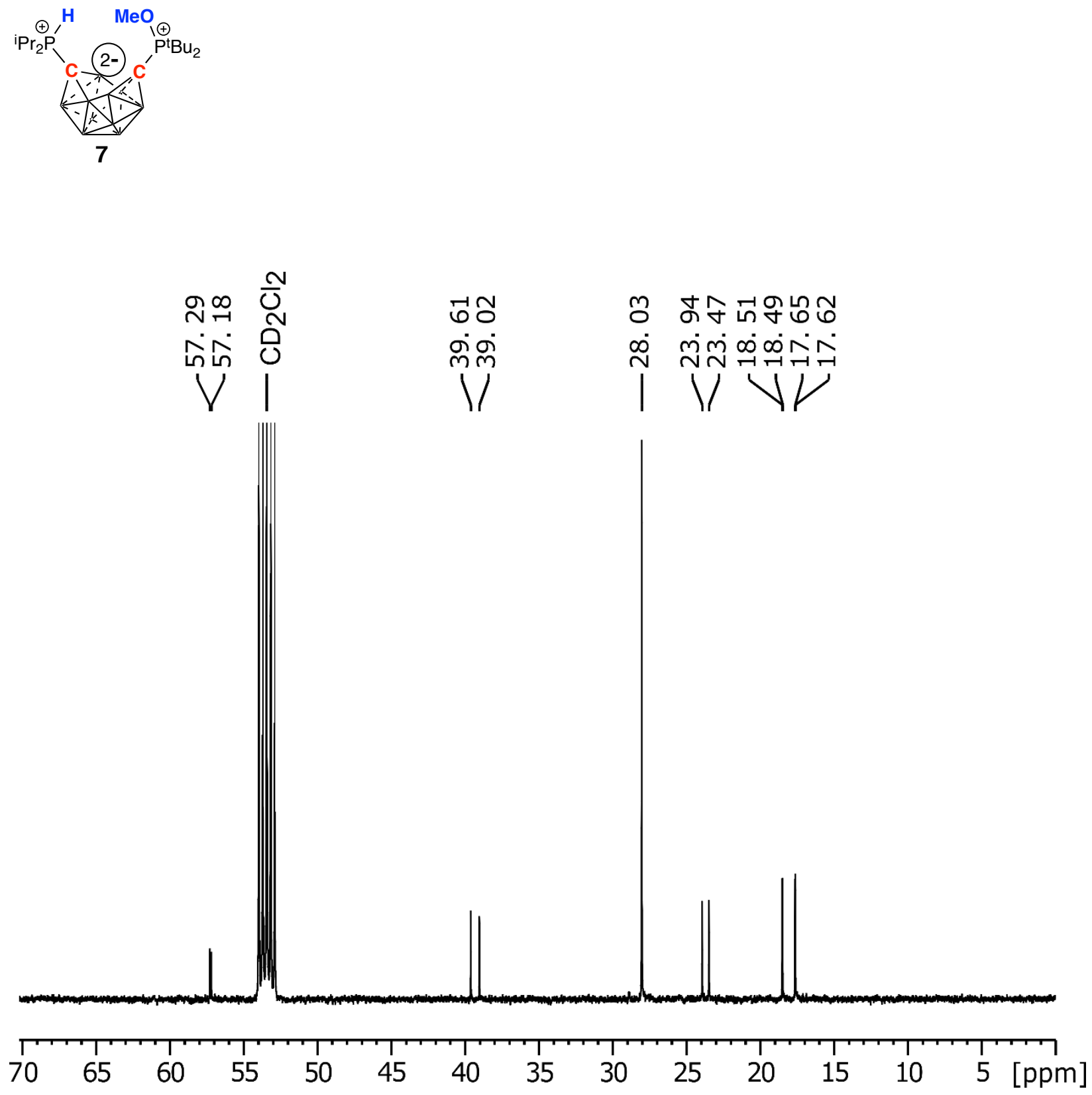

Figure S-23: The ${ }^{13} \mathrm{C}$ NMR spectrum of $\mathrm{P}^{\mathrm{t}} \mathrm{Bu}_{2}(\mathrm{OMe})-\mathrm{P}^{\mathrm{i}} \mathrm{Pr}_{2}(\mathrm{H})-\left(\mathrm{C}_{2} \mathrm{~B}_{10} \mathrm{H}_{10}\right)(7)$.

${ }^{13} \mathrm{C}$ NMR $\left(\mathrm{CD}_{2} \mathrm{Cl}_{2}\right): 57.2\left(\mathrm{~d},{ }^{2} J_{\mathrm{C}-\mathrm{P}}=11 \mathrm{~Hz}, \mathrm{OCH}_{3}\right), 39.3\left(\mathrm{~d},{ }^{1} J_{\mathrm{C}-\mathrm{P}}=59 \mathrm{~Hz}, \mathrm{P}\left(C\left(\mathrm{CH}_{3}\right)_{3}\right)_{2}\right), 28.0(\mathrm{~s}$, $\left.\mathrm{P}\left(\mathrm{C}\left(\mathrm{CH}_{3}\right)_{3}\right)_{2}\right), 23.7\left(\mathrm{~d},{ }^{1} J_{\mathrm{C}-\mathrm{P}}=47 \mathrm{~Hz}, \mathrm{P}\left(\mathrm{CH}\left(\mathrm{CH}_{3}\right)_{2}\right)_{2}\right), 18.5\left(\mathrm{~d},{ }^{2} J_{\mathrm{C}-\mathrm{P}}=3 \mathrm{~Hz}, \mathrm{P}\left(\mathrm{CH}\left(\mathrm{CH}_{3}\right)_{2}\right)_{2}\right), 17.6$ $\left(\mathrm{d},{ }^{2} J_{\mathrm{C}-\mathrm{P}}=3 \mathrm{~Hz}, \mathrm{P}\left(\mathrm{CH}\left(\mathrm{CH}_{3}\right)_{2}\right)_{2}\right)$. 


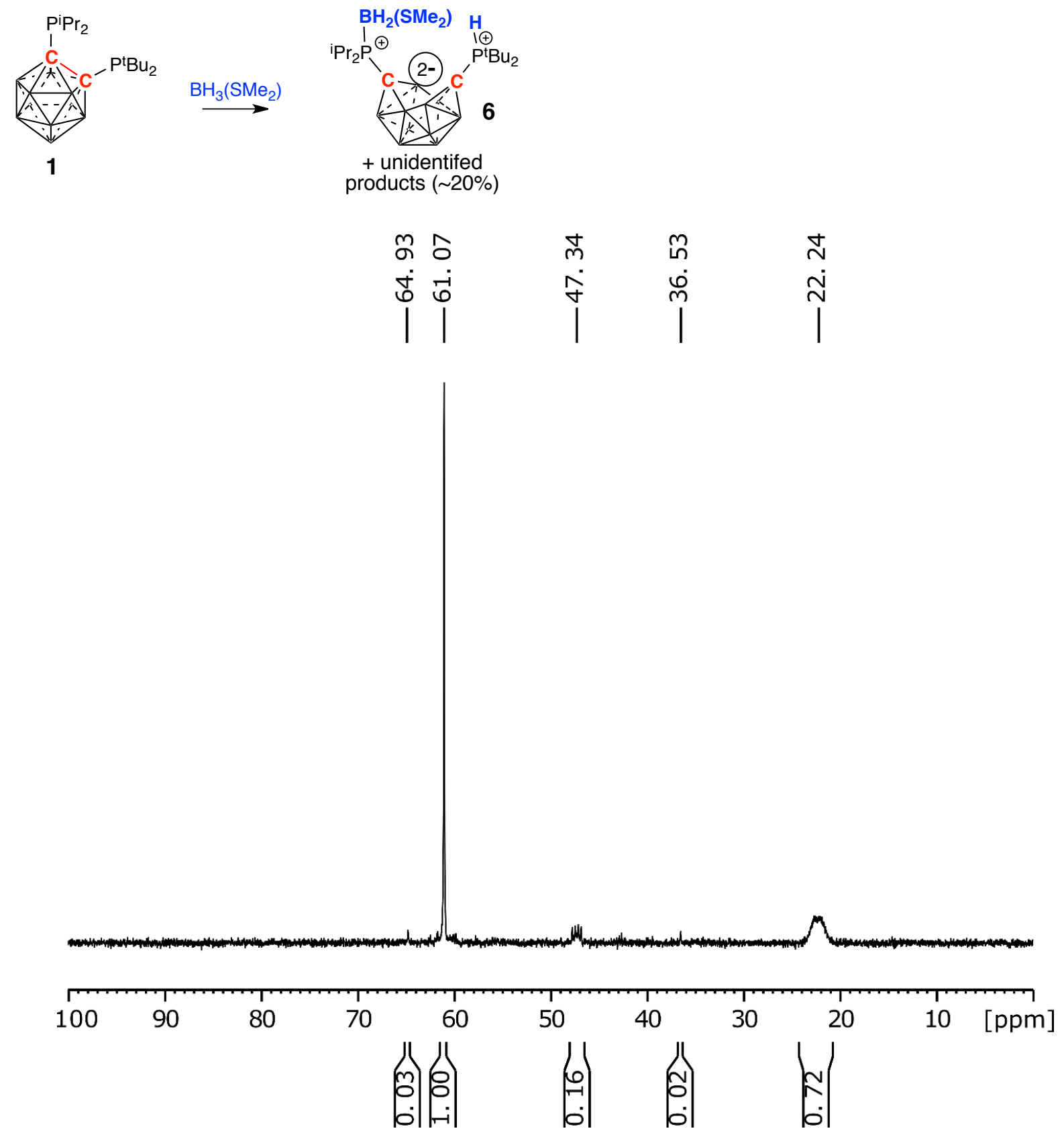

Figure S-24: The ${ }^{31} \mathrm{P}\left\{{ }^{1} \mathrm{H}\right\}$ NMR spectrum of the reaction mixture of 1 and $\mathrm{BH}_{3}\left(\mathrm{SMe}_{2}\right)$ after 30 min at room temperature. The major product of the reaction is $\mathrm{P}(\mathrm{H})^{\mathrm{t}} \mathrm{Bu}_{2}-\mathrm{P}\left(\mathrm{BH}_{2} \mathrm{SMe}_{2}\right)^{\mathrm{i}} \mathrm{Pr}_{2}-$ $\left(\mathrm{C}_{2} \mathrm{~B}_{10} \mathrm{H}_{10}\right)(\mathbf{6})$. 


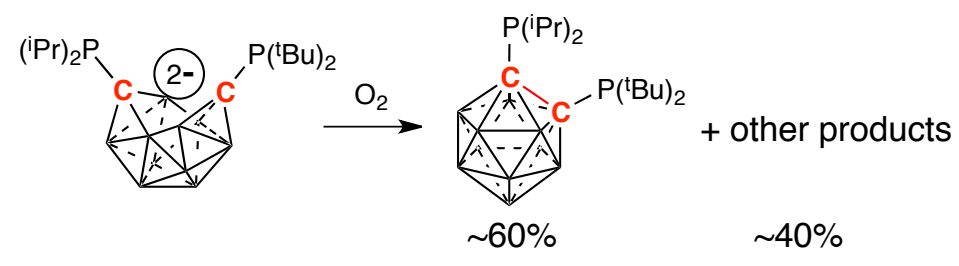

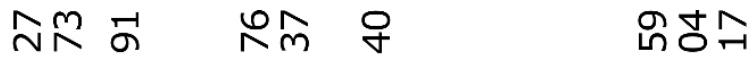

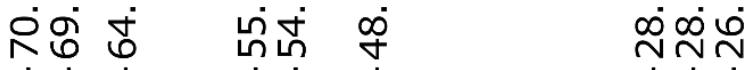

$$
\begin{aligned}
& \begin{array}{llll}
1 & 11
\end{array}
\end{aligned}
$$

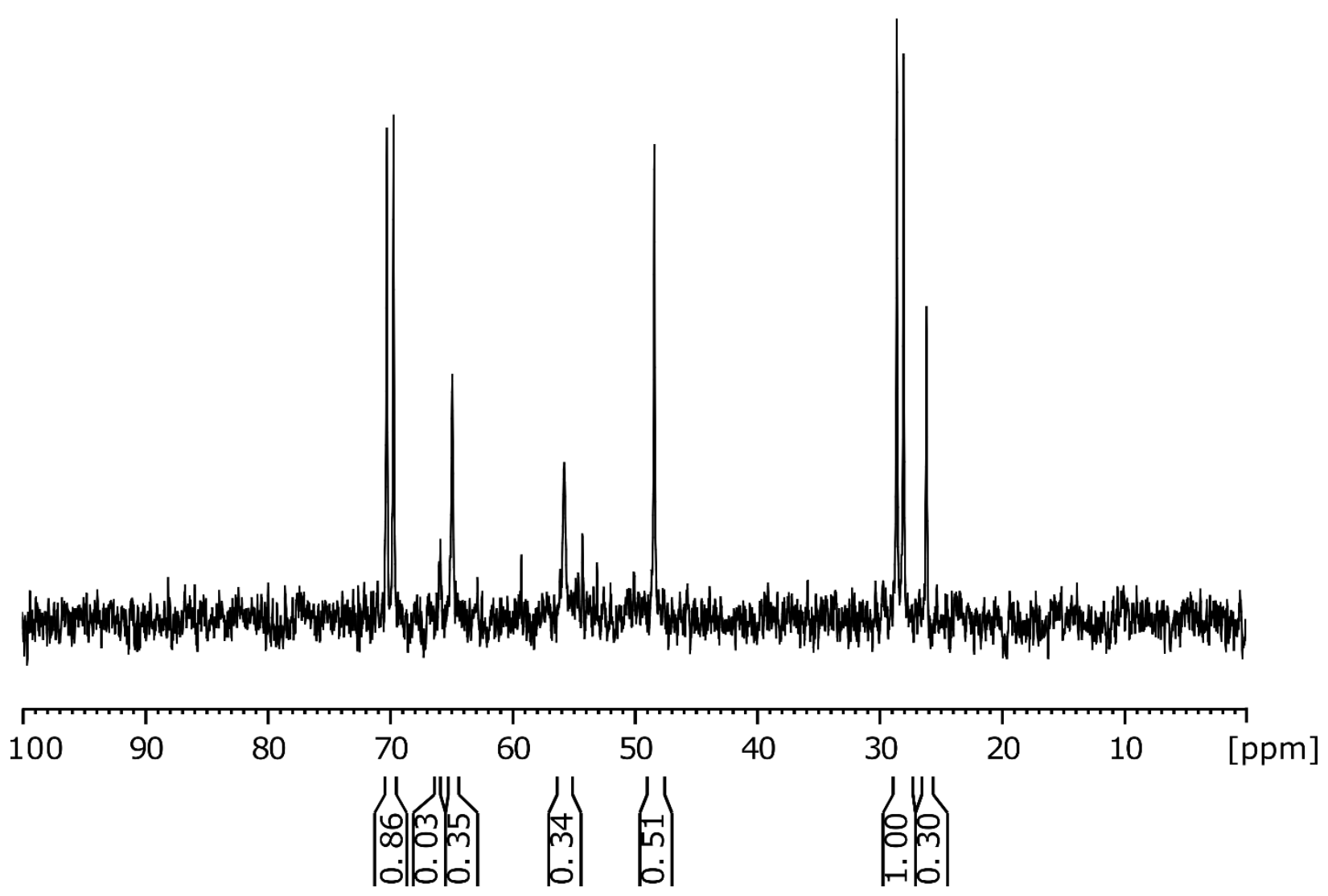

Figure S-25: The ${ }^{31} \mathrm{P}\left\{{ }^{1} \mathrm{H}\right\}$ NMR spectrum of the reaction mixture of $\mathrm{Na}_{2}(\mathrm{THF})_{6}\left[\mathrm{P}\left({ }^{\mathrm{t}} \mathrm{Bu}_{2}\right) \mathrm{P}\left({ }^{(} \mathrm{Pr}_{2}\right)\left(\mathrm{C}_{2} \mathrm{~B}_{10} \mathrm{H}_{10}\right)\right]$ (2) and dry $\mathrm{O}_{2}$ in THF at room temperature. The set of doublets at $28.1 \mathrm{ppm}$ and $69.9 \mathrm{ppm}$ corresponds to the parent $1-\mathrm{P}\left({ }^{\mathrm{t}} \mathrm{Bu}_{2}\right)-2-\mathrm{P}\left({ }^{\mathrm{i}} \mathrm{Pr}_{2}\right)-\left(\mathrm{C}_{2} \mathrm{~B}_{10} \mathrm{H}_{10}\right)(\mathbf{1})$. Other products were not identified. 


\section{Cyclic voltammetry analysis results}

Voltammograms were collected with Pine Research WaveNowXV 10V potentiostat. A cell equipped with a $3 \mathrm{~mm}$ dia. glassy carbon working electrode, a platinum wire as a counter electrode and a silver wire as pseudoreference electrode, referenced against a decamethylferrocene redox event $\left(\mathrm{Fc}_{2}{ }_{2}{ }^{+} / \mathrm{Fc}^{*}{ }_{2}\right)$ before and after measurements, was used. Tetrabutylammonium hexafluorophosphate was used as a supporting electrolyte. Tetrahydrofuran was used as a solvent.

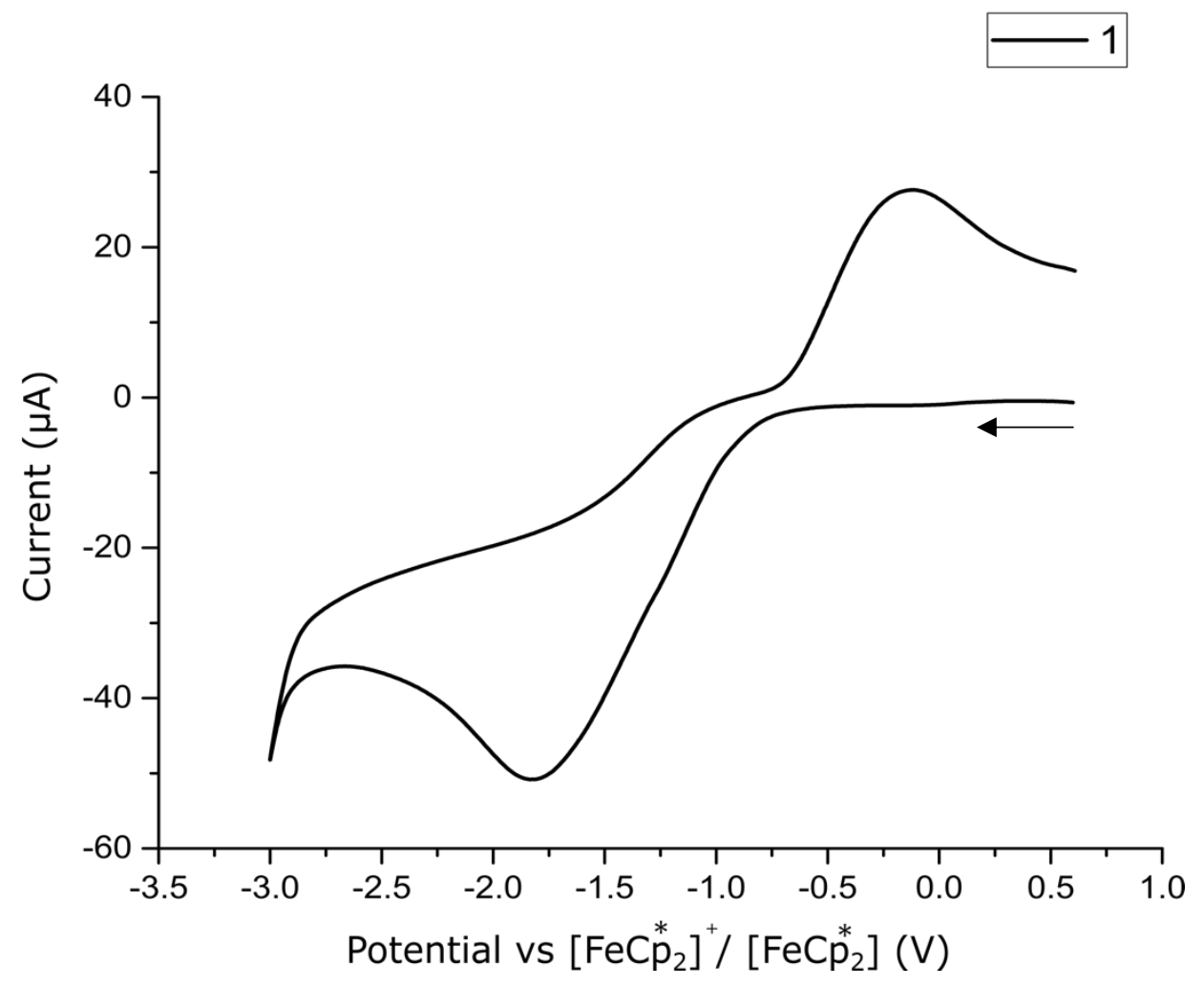

Figure S-26: Cyclic voltammogram of $5 \mathrm{mM}$ solution of 1-P'Buz-2- $\mathrm{P}^{\mathrm{i}} \mathrm{Pr}_{2}-\left(\mathrm{C}_{2} \mathrm{~B}_{10} \mathrm{H}_{10}\right)$ (1) on a glassy carbon working electrode. (Scan rate $0.1 \mathrm{~V} / \mathrm{s}$, electrolyte - tetrabutylammonium hexafluorophosphate in THF). 


\section{Cyclic voltammetry analysis of 3}

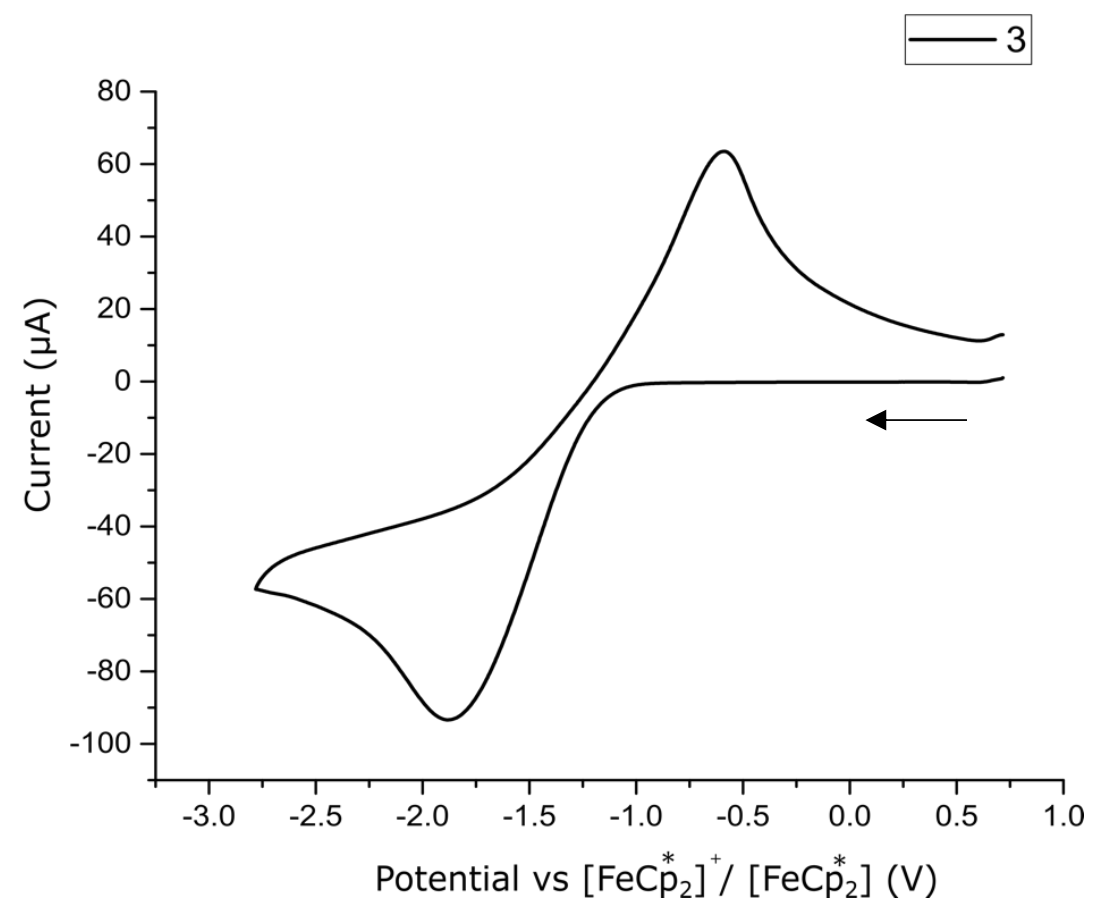

Figure S-27: The reduction of $5 \mathrm{mM}$ solution of $\mathrm{P}\left({ }^{\mathrm{i}} \mathrm{Pr}_{2}\right)_{2}-\left(\mathrm{C}_{2} \mathrm{~B}_{10} \mathrm{H}_{10}\right)(\mathbf{3})$ on a glassy carbon working electrode. (Scan rate $0.1 \mathrm{~V} / \mathrm{s}$, Electrolyte - tetrabutylammonium hexafluorophosphate in THF). 


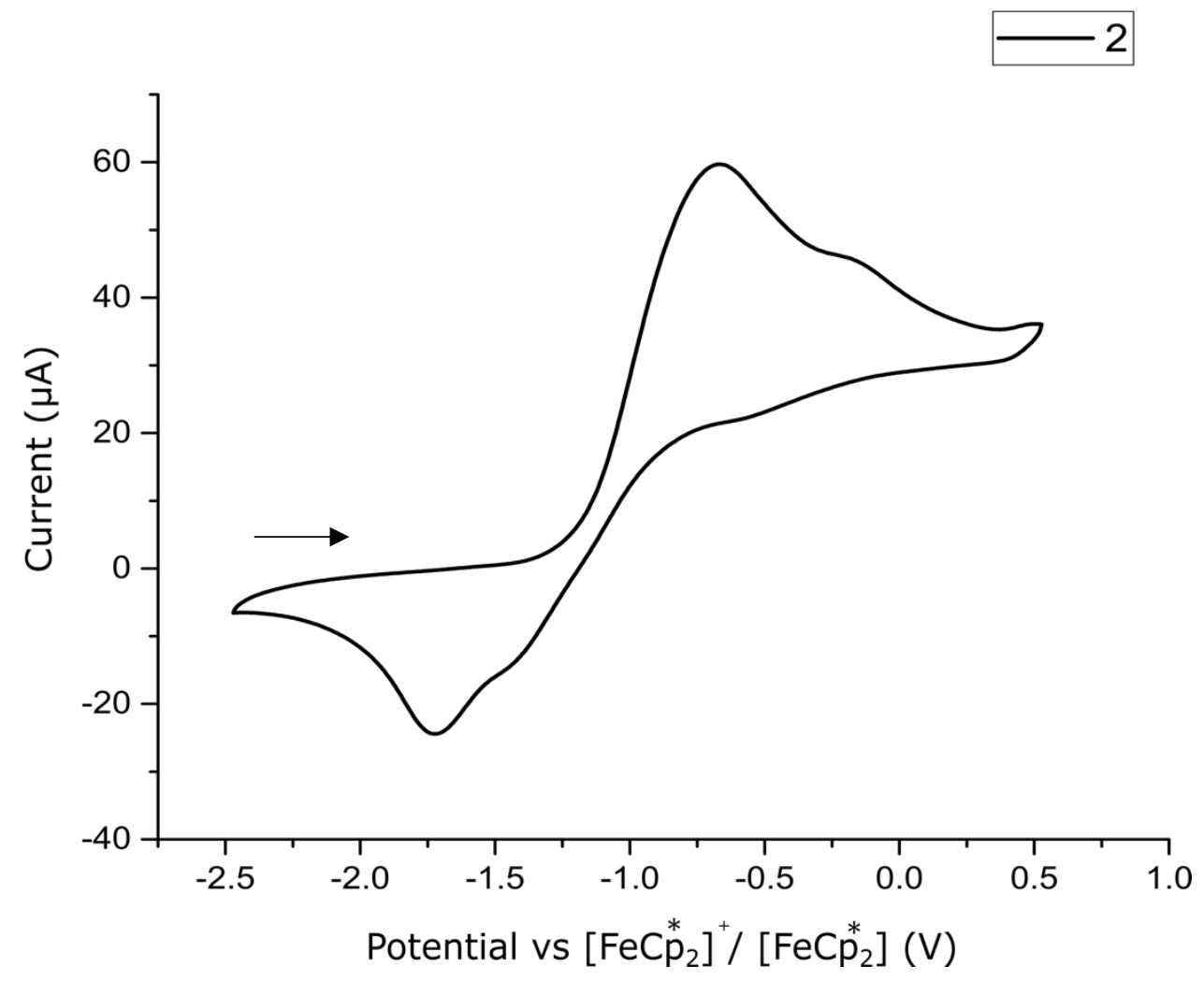

Figure S-28: Cyclic voltammogram of $5 \mathrm{mM}$ solution of $\mathrm{Na}_{2}\left[1-\mathrm{P}^{t} \mathrm{Bu}_{2}-2-\mathrm{P}^{\mathrm{i}} \mathrm{Pr}_{2}-\left(\mathrm{C}_{2} \mathrm{~B}_{10} \mathrm{H}_{10}\right)\right](2)$ on a glassy carbon working electrode. (Scan rate $0.1 \mathrm{~V} / \mathrm{s}$, electrolyte - tetrabutylammonium hexafluorophosphate in THF). 


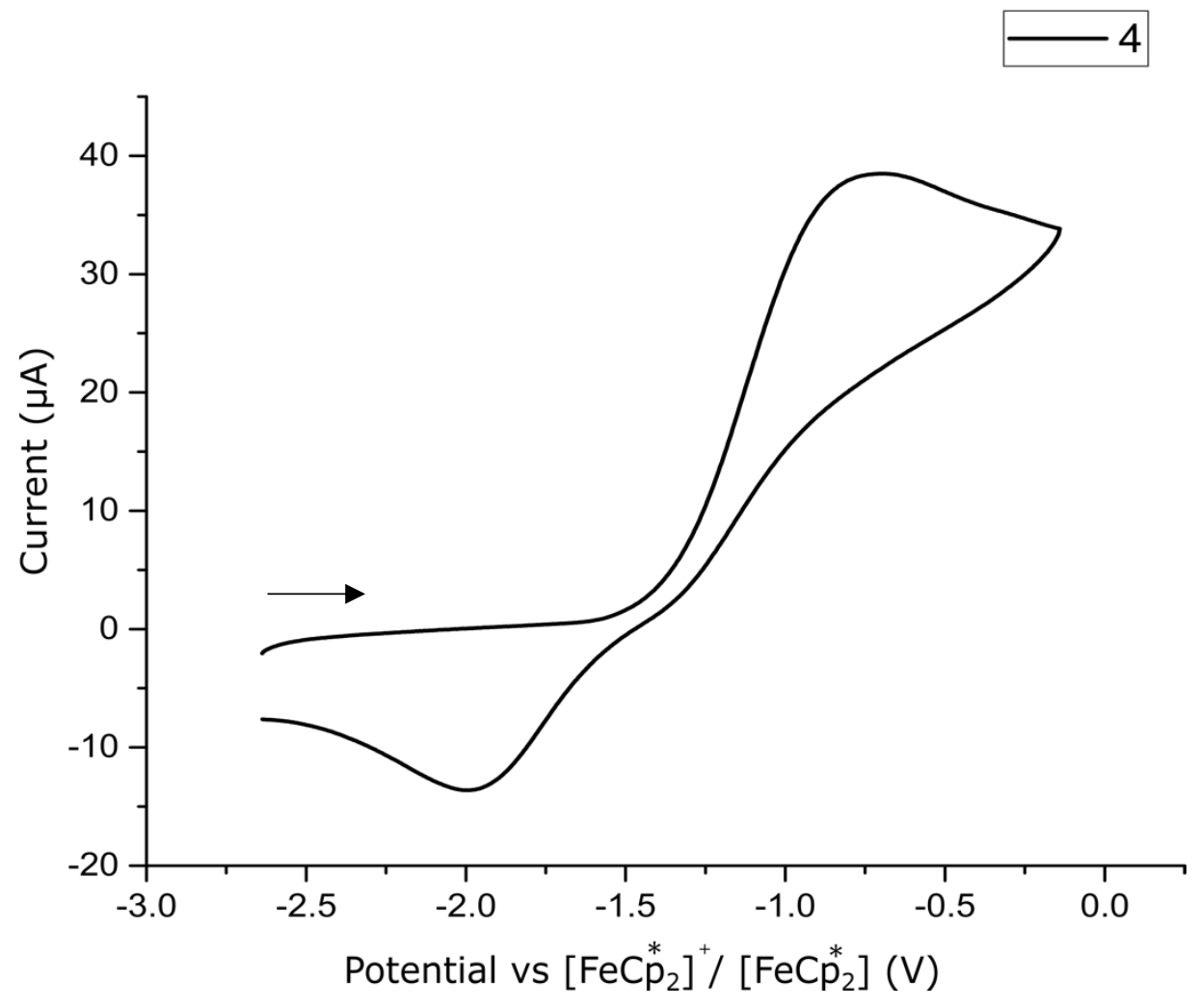

Figure S-29: Cyclic voltammogram of $5 \mathrm{mM}$ solution of $\mathrm{Na}_{2}\left[\mathrm{P}\left({ }^{\mathrm{i}} \mathrm{Pr}_{2}\right)_{2}-\left(\mathrm{C}_{2} \mathrm{~B}_{10} \mathrm{H}_{10}\right)\right](4)$ on a glassy carbon working electrode. (Scan rate $0.1 \mathrm{~V} / \mathrm{s}$, electrolyte - tetrabutylammonium hexafluorophosphate in THF). 


\section{X-ray single crystal structure determination details}

\section{X-Ray Structure Determination, 1-P'Buz-2-Pi $\operatorname{Pr}_{2}-\left(\mathrm{C}_{2} \mathbf{B}_{10} \mathrm{H}_{10}\right)$ (1)}

X-ray intensity data from a yellow needle were collected at 100(2) K using a Bruker D8 QUEST diffractometer equipped with a PHOTON-100 CMOS area detector and an Incoatec microfocus source (Mo K $\alpha$ radiation, $\lambda=0.71073 \AA$ ). The raw area detector data frames were reduced and corrected for absorption effects using the Bruker APEX3, SAINT+ and SADABS programs. ${ }^{1,2}$ Final unit cell parameters were determined by least-squares refinement of 9176 reflections taken from the data set. The structure was solved with SHELXT. ${ }^{3}$ Subsequent difference Fourier calculations and full-matrix least-squares refinement against $F^{2}$ were performed with SHELXL-2018 $8^{3}$ using OLEX $2 .^{4}$

The compound crystallizes in the orthorhombic system. The pattern of systematic absences in the intensity data was uniquely consistent with the space group $P 2_{1} 2_{1} 2_{1}$, which was confirmed by structure solution. The asymmetric unit consists of one molecule. All non-hydrogen atoms were refined with anisotropic displacement parameters. Hydrogen atoms bonded to carbon were located in difference Fourier maps before being placed in geometrically idealized positions and included as riding atoms with $d(\mathrm{C}-\mathrm{H})=1.00 \AA$ and $U$ iso $(\mathrm{H})=1.2 U$ eq $(\mathrm{C})$ for methine hydrogen atoms and $d(\mathrm{C}-\mathrm{H})=0.98 \AA$ and $U$ iso $(\mathrm{H})=1.5 U$ eq $(\mathrm{C})$ for methyl hydrogens. The methyl hydrogens were allowed to rotate as a rigid group to the orientation of maximum observed electron density. Hydrogen atoms bonded to boron were located and refined freely. The largest residual electron density peak in the final difference map is $0.28 \mathrm{e}^{-} / \AA^{3}$, located $0.71 \AA$ from C21. After the final refinement cycle the absolute structure (Flack) parameter was 0.004(18).

(1) APEX3 Version 2016.5-0 and SAINT+ Version 8.38A. Bruker AXS, Inc., Madison, Wisconsin, USA, 2016.

(2) SADABS-2016/2: Krause, L., Herbst-Irmer, R., Sheldrick G.M. and Stalke D. J. Appl. Cryst. 2015, 48, 3-10.

(3) (a) SHELXT: Sheldrick, G.M. Acta Cryst. 2015, A71, 3-8. (b) SHELXL: Sheldrick, G.M. Acta Cryst. 2015, C71, 3-8.

(4) OLEX2: a complete structure solution, refinement and analysis program. Dolomanov, O. V., Bourhis, L. J., Gildea, R. J., Howard J. A. K. and Puschmann, H. J. Appl. Cryst. 2009, 42, 339-341. 
Table S-1. Crystal data and structure refinement for 1-PtBu $2-2-\mathrm{P}^{\mathrm{i}} \mathrm{Pr}_{2}-\left(\mathrm{C}_{2} \mathrm{~B}_{10} \mathrm{H}_{10}\right)(\mathbf{1})$

Identification code

Empirical formula

Formula weight

Temperature/K

Crystal system

Space group

$\mathrm{a} / \AA$

$\mathrm{b} / \AA$

$\mathrm{c} / \AA$

$\alpha /{ }^{\circ}$

$\beta /{ }^{\circ}$

$\gamma /{ }^{\circ}$

Volume $/ \AA^{3}$

Z

$\rho_{\text {calc }} \mathrm{g} / \mathrm{cm}^{3}$

$\mu / \mathrm{mm}^{-1}$

$\mathrm{F}(000)$

Crystal size $/ \mathrm{mm}^{3}$

Radiation

$2 \Theta$ range for data collection/ ${ }^{\circ}$

Index ranges

Reflections collected

Independent reflections

Data/restraints/parameters

Goodness-of-fit on $\mathrm{F}^{2}$

Final $R$ indexes $[\mathrm{I}>=2 \sigma(\mathrm{I})]$

Final $\mathrm{R}$ indexes [all data]

Largest diff. peak/hole / e $\AA^{-3}$

Flack parameter
DER2009

$\mathrm{C}_{16} \mathrm{H}_{42} \mathrm{~B}_{10} \mathrm{P}_{2}$

404.53

100(2)

orthorhombic

$\mathrm{P} 2{ }_{1}{ }_{1}{ }_{1}$

10.6158(3)

14.2894(4)

15.8907(4)

90

90

90

2410.51(11)

4

1.115

0.182

872.0

$0.46 \times 0.28 \times 0.2$

$\operatorname{MoK} \alpha(\lambda=0.71073)$

4.614 to 65.33

$-16 \leq \mathrm{h} \leq 16,-21 \leq \mathrm{k} \leq 21,-24 \leq 1 \leq 23$

85541

$8828\left[\mathrm{R}_{\text {int }}=0.0564, \mathrm{R}_{\text {sigma }}=0.0299\right]$

$8828 / 0 / 303$

1.036

$\mathrm{R}_{1}=0.0285, \mathrm{wR}_{2}=0.0662$

$\mathrm{R}_{1}=0.0352, \mathrm{wR}_{2}=0.0689$

$0.28 /-0.23$

$0.004(18)$ 


\section{$\mathrm{X}$-Ray Structure Determination, [ $\left.\mathrm{Na}_{2}(\mathrm{THF})_{3}\left(\mathrm{C}_{14} \mathrm{H}_{38} \mathrm{~B}_{10} \mathrm{P}_{2}\right)\right]$}

( DER-219)

X-ray intensity data from a colorless plate were collected at 100(2) K using a Bruker D8 QUEST diffractometer equipped with a PHOTON-100 CMOS area detector and an Incoatec microfocus source (Mo K $\alpha$ radiation, $\lambda=0.71073 \AA$ ). The raw area detector data frames were reduced and corrected for absorption effects using the Bruker APEX3, SAINT+ and SADABS programs. ${ }^{1,2}$ Final unit cell parameters were determined by least-squares refinement of 9905 reflections taken from the data set. The structure was solved with SHELXT. ${ }^{3}$ Subsequent difference Fourier calculations and full-matrix least-squares refinement against $F^{2}$ were performed with SHELXL-2018 ${ }^{3}$ using OLEX2. ${ }^{4}$

The compound crystallizes in the triclinic system. The space group $P-1$ was confirmed by structure solution. The asymmetric unit consists of one formula unit. Two of the three THF molecules coordinated to sodium are disordered. They were modeled with either two (O2/C41$\mathrm{C} 44 \mathrm{~A} / \mathrm{B})$ or three $(\mathrm{O} 3 / \mathrm{C} 51-\mathrm{C} 54 \mathrm{~A} / \mathrm{B} / \mathrm{C})$ components. For each ligand, the oxygen atom is common to all components. The sum of the component occupancies was constrained to one for each group. 1,2- and 1.3- bond distances were restrained to be similar to those in the ordered THF (O1/C31C34) using SHELX SAME instructions. All non-hydrogen atoms were refined with anisotropic displacement parameters. Those of disordered atoms appearing nearly superimposed in the asymmetric unit were held equal (EADP). Hydrogen atoms bonded to carbon were placed in geometrically idealized positions and included as riding atoms with $d(\mathrm{C}-\mathrm{H})=1.00 \AA$ and $U$ iso $(\mathrm{H})$ $=1.2 U$ eq $(\mathrm{C})$ for methine hydrogen atoms, $d(\mathrm{C}-\mathrm{H})=0.99 \AA$ and $U$ iso $(\mathrm{H})=1.2 U$ eq $(\mathrm{C})$ for methylene hydrogen atoms, and $d(\mathrm{C}-\mathrm{H})=0.98 \AA$ and $U$ iso $(\mathrm{H})=1.5 \mathrm{Ueq}(\mathrm{C})$ for methyl hydrogens. The methyl hydrogens were allowed to rotate as a rigid group to the orientation of maximum observed electron density. Hydrogen atoms of the carborane cage were located in difference Fourier maps and refined freely. The largest residual electron density peak in the final difference map is $0.53 \mathrm{e}^{-} / \AA^{3}$, located $0.64 \AA$ from C43A.

(1) APEX3 Version 2016.5-0 and SAINT+ Version 8.38A. Bruker AXS, Inc., Madison, Wisconsin, USA, 2016.

(2) SADABS-2016/2: Krause, L., Herbst-Irmer, R., Sheldrick G.M. and Stalke D. J. Appl. Cryst. 2015, 48, 3-10.

(3) (a) SHELXT: Sheldrick, G.M. Acta Cryst. 2015, A71, 3-8. (b) SHELXL: Sheldrick, G.M. Acta Cryst. 2015, C71, 3-8.

(4) OLEX2: a complete structure solution, refinement and analysis program. Dolomanov, O. V., Bourhis, L. J., Gildea, R. J., Howard J. A. K. and Puschmann, H. J. Appl. Cryst. 2009, 42, 339-341. 
Table S-2. Crystal data and structure refinement for $\left[\mathrm{Na}_{2}(\mathrm{THF})_{3}\left(\mathrm{C}_{14} \mathrm{H}_{38} \mathrm{~B}_{10} \mathrm{P}_{2}\right)\right]$

Identification code

Empirical formula

Formula weight

Temperature/K

Crystal system

Space group

$\mathrm{a} / \AA$

$\mathrm{b} / \AA$

$\mathrm{c} / \AA$

$\alpha /{ }^{\circ}$

$\beta /{ }^{\circ}$

$\gamma /{ }^{\circ}$

Volume $/ \AA^{3}$

Z

$\rho_{\text {calc }} \mathrm{g} / \mathrm{cm}^{3}$

$\mu / \mathrm{mm}^{-1}$

$\mathrm{F}(000)$

Crystal size $/ \mathrm{mm}^{3}$

Radiation

$2 \Theta$ range for data collection/ ${ }^{\circ}$

Index ranges

Reflections collected

Independent reflections

Data/restraints/parameters

Goodness-of-fit on $\mathrm{F}^{2}$

Final $\mathrm{R}$ indexes [I $>=2 \sigma(\mathrm{I})]$

Final $\mathrm{R}$ indexes [all data]

Largest diff. peak/hole / e $\AA^{-3}$
DER219

$\mathrm{C}_{26} \mathrm{H}_{62} \mathrm{~B}_{10} \mathrm{Na}_{2} \mathrm{O}_{3} \mathrm{P}_{2}$

638.77

100(2)

triclinic

P-1

$11.5195(4)$

13.0933(4)

14.0951(5)

$70.4830(10)$

$76.735(2)$

$72.718(2)$

1893.20(11)

2

1.121

0.164

688.0

$0.28 \times 0.2 \times 0.06$

$\operatorname{MoK} \alpha(\lambda=0.71073)$

4.41 to 56.702

$-15 \leq \mathrm{h} \leq 15,-17 \leq \mathrm{k} \leq 17,-18 \leq 1 \leq 18$

78628

$9446\left[\mathrm{R}_{\text {int }}=0.0483, \mathrm{R}_{\text {sigma }}=0.0339\right]$

$9446 / 152 / 488$

1.029

$\mathrm{R}_{1}=0.0445, \mathrm{wR}_{2}=0.1035$

$\mathrm{R}_{1}=0.0652, \mathrm{wR}_{2}=0.1145$

$0.53 /-0.55$ 


\section{$X$-Ray Structure Determination, $\mathrm{P}(\mathrm{H})^{\mathrm{t}} \mathrm{Bu}_{2}-\mathrm{P}(\mathrm{H})^{\mathrm{i}} \mathrm{Pr}_{2}-\left(\mathrm{C}_{2} \mathrm{~B}_{10} \mathrm{H}_{10}\right)(5)$}

X-ray intensity data from colorless block were collected at 100(2) K using a Bruker D8 QUEST diffractometer equipped with a PHOTON-100 CMOS area detector and an Incoatec microfocus source (Mo K $\alpha$ radiation, $\lambda=0.71073 \AA$ ). The raw area detector data frames were reduced and corrected for absorption effects using the Bruker APEX3, SAINT+ and SADABS programs. ${ }^{1,2}$ The structure was solved with SHELXT. ${ }^{3}$ Subsequent difference Fourier calculations and full-matrix least-squares refinement against $F^{2}$ were performed with SHELXL-2018 $8^{3}$ using OLEX2. ${ }^{4}$

The compound crystallizes in the monoclinic system. The pattern of systematic absences in the intensity data was consistent with the space group $P 2_{1} / c$, which was confirmed by structure solution. The asymmetric unit consists of one molecule. All non-hydrogen atoms were refined with anisotropic displacement parameters. All hydrogen atoms were located in difference Fourier maps. Those bonded to carbon were placed in geometrically idealized positions and included as riding atoms with $d(\mathrm{C}-\mathrm{H})=1.00 \AA$ and $U \mathrm{iso}(\mathrm{H})=1.2 U \mathrm{eq}(\mathrm{C})$ for methine hydrogen atoms and $d(\mathrm{C}-\mathrm{H})=$ $0.98 \AA$ and $U$ iso $(\mathrm{H})=1.5 U$ eq $(\mathrm{C})$ for methyl hydrogens. The methyl hydrogens were allowed to rotate as a rigid group to the orientation of maximum observed electron density. Hydrogen atoms bonded to boron and phosphorus were refined freely. The largest residual electron density peak in the final difference map is $0.35 \mathrm{e}^{-} / \AA^{3}$, located $0.85 \AA$ from $\mathrm{C} 24$.

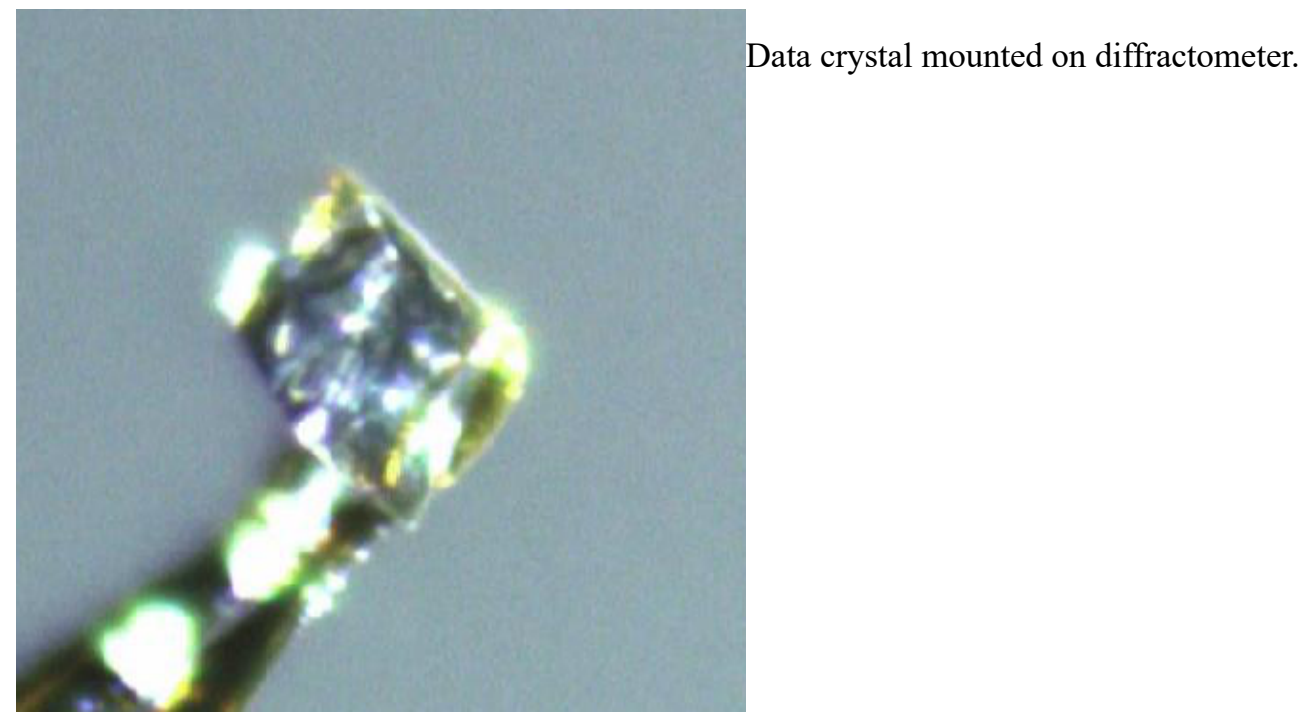

(1) APEX3 Version 2018.1-0 and SAINT+ Version 8.38A. Bruker AXS, Inc., Madison, Wisconsin, USA, 2016.

(2) SADABS-2016/2: Krause, L., Herbst-Irmer, R., Sheldrick G.M. and Stalke D. J. Appl. Cryst. 2015, 48, 3-10.

(3) (a) SHELXT: Sheldrick, G.M. Acta Cryst. 2015, A71, 3-8. (b) SHELXL: Sheldrick, G.M. Acta Cryst. 2015, C71, 3-8.

(4) OLEX2: a complete structure solution, refinement and analysis program. Dolomanov, O. V., Bourhis, L. J., Gildea, R. J., Howard J. A. K. and Puschmann, H. J. Appl. Cryst. 2009, 42, 339-341. 
Table S-3. Crystal data and structure refinement for $\mathrm{P}(\mathrm{H})^{\mathrm{t}} \mathrm{Bu}_{2}-\mathrm{P}(\mathrm{H})^{i} \mathrm{Pr}_{2}-\left(\mathrm{C}_{2} \mathrm{~B}_{10} \mathrm{H}_{10}\right)$ (5)

Identification code

Empirical formula

Formula weight

Temperature/K

Crystal system

Space group

$\mathrm{a} / \AA \AA$

$\mathrm{b} / \AA$

$\mathrm{c} / \AA$

$\alpha /^{\circ}$

$\beta /{ }^{\circ}$

$\gamma /{ }^{\circ}$

Volume $/ \AA^{3}$

Z

$\rho_{\text {calc }} \mathrm{g} / \mathrm{cm}^{3}$

$\mu / \mathrm{mm}^{-1}$

$\mathrm{F}(000)$

Crystal size $/ \mathrm{mm}^{3}$

Radiation

$2 \Theta$ range for

Index ranges

Reflections collected

Independent reflections

Data/restraints/parameters

Goodness-of-fit on $\mathrm{F}^{2}$

Final $\mathrm{R}$ indexes $[\mathrm{I}>=2 \sigma(\mathrm{I})]$

Final R indexes [all data]

Largest diff. peak/hole / e $\AA^{-3} \quad 0.35 /-0.30$ gb1120

$\mathrm{C}_{16} \mathrm{H}_{44} \mathrm{~B}_{10} \mathrm{P}_{2}$

406.55

100(2)

monoclinic

$\mathrm{P} 2{ }_{1} / \mathrm{c}$

11.7683(7)

$13.9233(8)$

15.6885(9)

90

107.325(3)

90

2454.0(2)

4

1.100

0.179

880.0

$0.18 \times 0.14 \times 0.12$

$\operatorname{MoK} \alpha(\lambda=0.71073)$

$-16 \leq \mathrm{h} \leq 16,-19 \leq \mathrm{k} \leq 19,-22 \leq 1 \leq 22$

115495

$7100\left[\mathrm{R}_{\text {int }}=0.0611, \mathrm{R}_{\text {sigma }}=0.0230\right]$

$7100 / 0 / 312$

1.041

$\mathrm{R}_{1}=0.0316, \mathrm{wR}_{2}=0.0779$

$\mathrm{R}_{1}=0.0426, \mathrm{wR}_{2}=0.0836$ 


\section{$\mathrm{X}$-Ray Structure Determination, $\mathbf{P}\left(\mathrm{BH}_{2} \mathrm{SMe}_{2}\right)^{\mathrm{i}} \mathrm{Pr}_{2}-\mathrm{P}(\mathrm{H})^{\mathrm{t}} \mathrm{Bu}_{2}-\left(\mathrm{C}_{2} \mathrm{~B}_{10} \mathrm{H}_{10}\right)$ (6) ( gb4082a)}

X-ray intensity data from a colorless needle were collected at 100(2) K using a Bruker D8 QUEST diffractometer equipped with a PHOTON-II area detector and an Incoatec microfocus source (Mo K $\alpha$ radiation, $\lambda=0.71073 \AA$ ). The raw area detector data frames were reduced, scaled and corrected for absorption effects using the Bruker APEX3, SAINT+ and SADABS programs. ${ }^{1,2}$ The structure was solved with SHELXT. ${ }^{3}$ Subsequent difference Fourier calculations and fullmatrix least-squares refinement against $F^{2}$ were performed with SHELXL-2018 ${ }^{3}$ using OLEX2. ${ }^{4}$

The compound crystallizes in the monoclinic system. The pattern of systematic absences in the intensity data was consistent with the space group $P 2_{1} / n$. The asymmetric unit consists of one molecule. All non-hydrogen atoms were refined with anisotropic displacement parameters. Hydrogen atoms bonded to carbon were located in difference Fourier maps before being placed in geometrically idealized positions and included as riding atoms with $d(\mathrm{C}-\mathrm{H})=1.00 \AA$ and $U$ iso $(\mathrm{H})$ $=1.2 U \mathrm{eq}(\mathrm{C})$ for methine hydrogen atoms and $d(\mathrm{C}-\mathrm{H})=0.98 \AA$ and $U$ iso $(\mathrm{H})=1.5 \mathrm{Ueq}(\mathrm{C})$ for methyl hydrogens. The methyl hydrogens were allowed to rotate as a rigid group to the orientation of maximum observed electron density. All hydrogen atoms bonded to phosphorus and boron were located and refined freely. The largest residual electron density peak in the final difference map is $0.45 \mathrm{e}^{-} / \AA^{3}$, located $0.83 \AA$ from P1.

(1) APEX3 Version 2019.1-0 and SAINT+ Version 8.40A. Bruker Nano, Inc., Madison, WI, USA, 2019.

(2) SADABS-2016/2: Krause, L., Herbst-Irmer, R., Sheldrick G.M. and Stalke D. J. Appl. Cryst. 2015, 48, 3-10.

(3) (a) SHELXT: Sheldrick, G.M. Acta Cryst. 2015, A71, 3-8. (b) SHELXL: Sheldrick, G.M. Acta Cryst. 2015, C71, 3-8.

(4) OLEX2: a complete structure solution, refinement and analysis program. Dolomanov, O. V., Bourhis, L. J., Gildea, R. J., Howard J. A. K. and Puschmann, H. J. Appl. Cryst. 2009, 42, 339-341. 
Table S-4. Crystal data and structure refinement for $\mathrm{P}\left(\mathrm{BH}_{2} \mathrm{SMe}_{2}\right)\left({ }^{\mathrm{i}} \mathrm{Pr}_{2}\right) \mathrm{P}\left({ }^{(} \mathrm{Bu}_{2}\right)(\mathrm{H})\left(\mathrm{C}_{2} \mathrm{~B}_{10} \mathrm{H}_{10}\right)(6)$

Identification code gb4082a_needle

Empirical formula

$\mathrm{C}_{18} \mathrm{H}_{51} \mathrm{~B}_{11} \mathrm{P}_{2} \mathrm{~S}$

Formula weight

480.49

Temperature/K

$100(2)$

Crystal system

monoclinic

Space group

$\mathrm{P} 21 / \mathrm{n}$

$\mathrm{a} / \AA$

9.5577(4)

$\mathrm{b} / \AA$

17.8459(7)

$\mathrm{c} / \AA$

$17.1715(7)$

$\alpha /{ }^{\circ}$

90

$\beta /{ }^{\circ}$

$98.750(2)$

$\gamma /{ }^{\circ}$

90

Volume $/ \AA^{3}$

2894.8(2)

Z

4

$\rho_{\text {calcg }} / \mathrm{cm}^{3}$

1.103

$\mu / \mathrm{mm}^{-1}$

0.230

$\mathrm{F}(000)$

1040.0

Crystal size $/ \mathrm{mm}^{3}$

$0.28 \times 0.04 \times 0.04$

Radiation

$\operatorname{MoK} \alpha(\lambda=0.71073)$

$2 \Theta$ range for data collection/ ${ }^{\circ}$

Index ranges

4.564 to 55.032

Reflections collected

$-11 \leq \mathrm{h} \leq 12,-23 \leq \mathrm{k} \leq 23,-22 \leq 1 \leq 22$

Independent reflections

54181

Data/restraints/parameters

$6649\left[\mathrm{R}_{\text {int }}=0.0495, \mathrm{R}_{\text {sigma }}=0.0287\right]$

Goodness-of-fit on $\mathrm{F}^{2}$ $6649 / 0 / 353$

Final R indexes $[\mathrm{I}>=2 \sigma(\mathrm{I})]$

1.034

Final R indexes [all data]

$\mathrm{R}_{1}=0.0398, \mathrm{wR}_{2}=0.0951$

Largest diff. peak/hole / e $\AA^{-3}$ $\mathrm{R}_{1}=0.0497, \mathrm{wR}_{2}=0.1005$

$0.45 /-0.26$ 


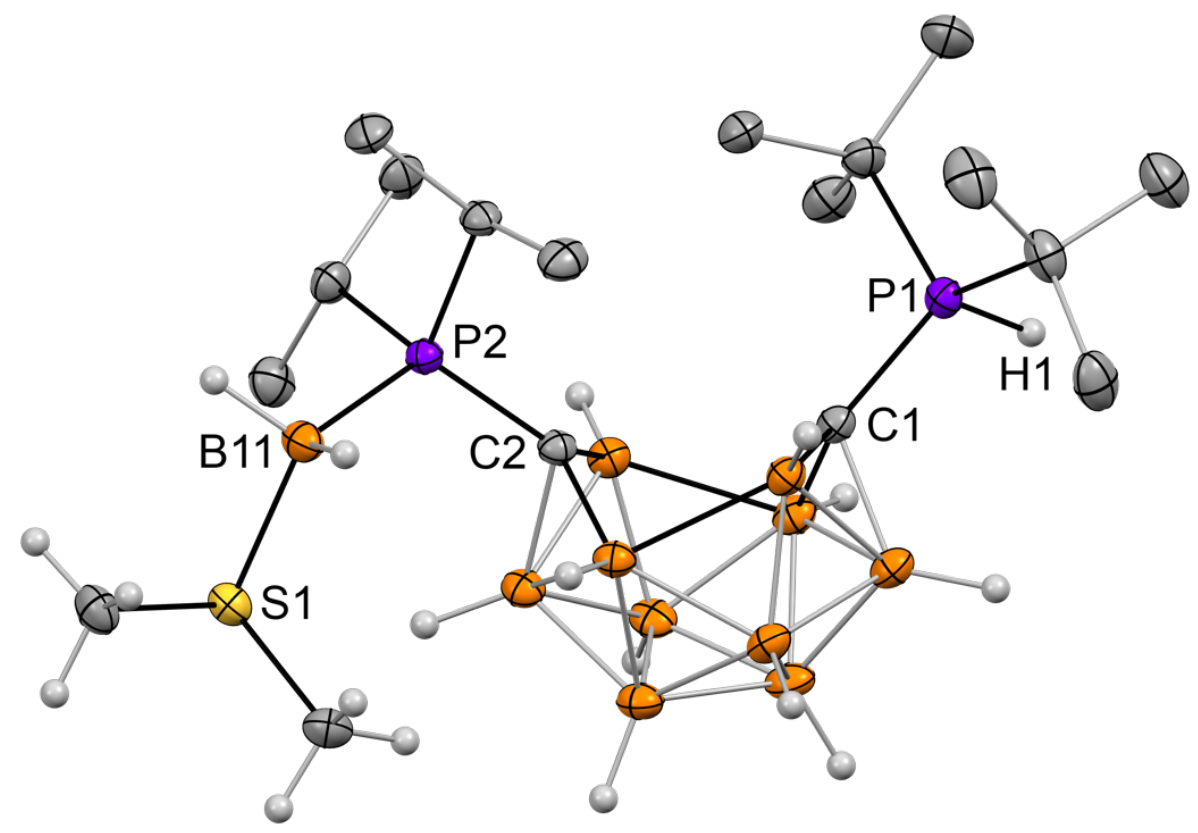

Figure S-30. The displacement ellipsoid plot (50\% probability) of the major product on the reaction of 1 and $\mathrm{BH}_{3} \mathrm{SMe}_{2}$ in THF at room temperature: $\mathrm{P}\left(\mathrm{BH}_{2} \mathrm{SMe}_{2}\right)\left({ }^{\mathrm{i}} \mathrm{Pr}_{2}\right)-\mathrm{P}\left({ }^{\mathrm{t}} \mathrm{Bu}_{2}\right)(\mathrm{H})-\left(\mathrm{C}_{2} \mathrm{~B}_{10} \mathrm{H}_{10}\right)(6)$. Hydrogen atoms of alkyl groups are not shown. 


\section{X-Ray Structure Determination, $\mathrm{P}(\mathrm{H})^{\mathrm{i}} \mathrm{Pr}_{2}-\mathrm{P}^{\mathrm{t}} \mathrm{Bu}_{2}(\mathrm{OMe})-\left(\mathrm{C}_{2} \mathrm{~B}_{10} \mathrm{H}_{10}\right)(7)$}

X-ray intensity data from a colorless prismatic rod were collected at 100(2) K using a Bruker D8 QUEST diffractometer equipped with a PHOTON-100 CMOS area detector and an Incoatec microfocus source (Mo K $\alpha$ radiation, $\lambda=0.71073 \AA$ ). The raw area detector data frames were reduced and corrected for absorption effects using the Bruker APEX3, SAINT+ and SADABS programs. ${ }^{1,2}$ The structure was solved with SHELXT. ${ }^{3}$ Subsequent difference Fourier calculations and full-matrix least-squares refinement against $F^{2}$ were performed with SHELXL-2018 ${ }^{3}$ using OLEX2. ${ }^{4}$

The compound crystallizes in the monoclinic system. The pattern of systematic absences in the intensity data was consistent with the space group $P 2_{1} / c$, which was confirmed by structure solution. The asymmetric unit consists of one molecule. All non-hydrogen atoms were refined with anisotropic displacement parameters. All hydrogen atoms were located in difference Fourier maps. Those bonded to carbon were placed in geometrically idealized positions and included as riding atoms with $d(\mathrm{C}-\mathrm{H})=1.00 \AA$ and $U \operatorname{iso}(\mathrm{H})=1.2 U \mathrm{eq}(\mathrm{C})$ for methine hydrogen atoms and $d(\mathrm{C}-\mathrm{H})=$ $0.98 \AA$ and $\operatorname{Uiso}(\mathrm{H})=1.5 U \mathrm{eq}(\mathrm{C})$ for methyl hydrogens. The methyl hydrogens were allowed to rotate as a rigid group to the orientation of maximum observed electron density. Hydrogen atoms bonded to boron and phosphorus were refined freely. The largest residual electron density peak in the final difference map is $0.38 \mathrm{e}^{-} / \AA^{3}$, located $0.86 \AA$ from $\mathrm{C} 1$.

(1) APEX3 Version 2018.1-0 and SAINT+ Version 8.38A. Bruker AXS, Inc., Madison, Wisconsin, USA, 2016.

(2) SADABS-2016/2: Krause, L., Herbst-Irmer, R., Sheldrick G.M. and Stalke D. J. Appl. Cryst. 2015, 48, 3-10.

(3) (a) SHELXT: Sheldrick, G.M. Acta Cryst. 2015, A71, 3-8. (b) SHELXL: Sheldrick, G.M. Acta Cryst. 2015, C71, 3-8.

(4) OLEX2: a complete structure solution, refinement and analysis program. Dolomanov, O. V., Bourhis, L. J., Gildea, R. J., Howard J. A. K. and Puschmann, H. J. Appl. Cryst. 2009, 42, 339-341. 
Table S-5. Crystal data and structure refinement for $\mathrm{P}(\mathrm{H})^{\mathrm{i}} \mathrm{Pr}_{2}-\mathrm{P}^{\mathrm{t}} \mathrm{Bu}_{2}(\mathrm{OMe})-\left(\mathrm{C}_{2} \mathrm{~B}_{10} \mathrm{H}_{10}\right)(7)$

\begin{tabular}{|c|c|}
\hline Identification code & gb2016 \\
\hline Empirical formula & $\mathrm{C}_{17} \mathrm{H}_{46} \mathrm{~B}_{10} \mathrm{OP}_{2}$ \\
\hline Formula weight & 436.58 \\
\hline Temperature/K & $100(2)$ \\
\hline Crystal system & monoclinic \\
\hline Space group & $\mathrm{P} 2_{1} / \mathrm{c}$ \\
\hline $\mathrm{a} / \AA \AA$ & $11.7264(6)$ \\
\hline $\mathrm{b} / \AA$ & $12.5723(6)$ \\
\hline $\mathrm{c} / \AA ̊$ & $17.8240(9)$ \\
\hline$\alpha /{ }^{\circ}$ & 90 \\
\hline$\beta /{ }^{\circ}$ & $101.452(2)$ \\
\hline$\gamma /{ }^{\circ}$ & 90 \\
\hline Volume $/ \AA^{3}$ & $2575.4(2)$ \\
\hline Z & 4 \\
\hline$\rho_{\text {calc }} \mathrm{g} / \mathrm{cm}^{3}$ & 1.126 \\
\hline$\mu / \mathrm{mm}^{-1}$ & 0.177 \\
\hline $\mathrm{F}(000)$ & 944.0 \\
\hline Crystal size $/ \mathrm{mm}^{3}$ & $0.28 \times 0.2 \times 0.15$ \\
\hline Radiation & $\operatorname{MoK} \alpha(\lambda=0.71073)$ \\
\hline $2 \Theta$ range for data collection/ ${ }^{\circ}$ & 3.992 to 65.104 \\
\hline Index ranges & $-17 \leq \mathrm{h} \leq 17,-19 \leq \mathrm{k} \leq 19,-26 \leq 1 \leq 26$ \\
\hline Reflections collected & 126233 \\
\hline Independent reflections & $9261\left[\mathrm{R}_{\mathrm{int}}=0.0534, \mathrm{R}_{\text {sigma }}=0.0244\right]$ \\
\hline Data/restraints/parameters & $9261 / 0 / 327$ \\
\hline Goodness-of-fit on $\mathrm{F}^{2}$ & 1.032 \\
\hline Final R indexes $[I>=2 \sigma(I)]$ & $\mathrm{R}_{1}=0.0296, \mathrm{wR}_{2}=0.0795$ \\
\hline Final $\mathrm{R}$ indexes [all data] & $\mathrm{R}_{1}=0.0381, \mathrm{wR}_{2}=0.0839$ \\
\hline Largest diff. peak/hole / e $\AA^{-3}$ & $0.38 /-0.27$ \\
\hline
\end{tabular}




\section{Computational details.}

The density functional theory calculations were carried out utilizing SCM ADF software package. ${ }^{2}$ Molecular geometry was optimized based on the atomic coordinates from the X-ray single-crystal diffraction data using the hybrid PBE0 functional and the TZP basis set with scalar relativistic effects considered using the ZORA formalism. Frequency calculations were carried out to confirm the local minimum results.

Table S-6. The optimized geometry coordinates for 1-P $\mathrm{Bu}_{2}-2-\mathrm{P}^{\mathrm{i}} \mathrm{Pr}_{2}-\left(\mathrm{C}_{2} \mathrm{~B}_{10} \mathrm{H}_{10}\right)(\mathbf{1})$

$\begin{array}{lcrrl}\text { Atom } & \mathrm{X} & \mathrm{Y} & \mathrm{Z} & \text { (Angstrom) } \\ \text { 1.P } & 7.938721 & 2.239895 & 4.234957 \\ \text { 2.P } & 5.365806 & 2.372073 & 6.392998 \\ \text { 3.C } & 6.226564 & 1.925167 & 3.431098 \\ \text { 4.C } & 4.855745 & 2.106439 & 4.596498 \\ \text { 5.C } & 8.740245 & 0.509937 & 4.517282 \\ \text { 6.C } & 8.222753 & 0.036212 & 5.880630 \\ \text { 7.H } & 8.453036 & 0.761855 & 6.662650 \\ \text { 8.H } & 8.710221 & -0.911689 & 6.131002 \\ \text { 9.H } & 7.149499 & -0.129457 & 5.891734 \\ \text { 10.C } & 10.252033 & 0.682586 & 4.687666 \\ \text { 11.H } & 10.761273 & 0.845780 & 3.738961 \\ \text { 12.H } & 10.644307 & -0.247298 & 5.110169 \\ \text { 13.H } & 10.508465 & 1.488427 & 5.377341 \\ \text { 14.C } & 8.515862 & -0.576522 & 3.470618 \\ \text { 15.H } & 7.479718 & -0.900927 & 3.418541 \\ \text { 16.H } & 9.117036 & -1.450220 & 3.745132 \\ \text { 17.H } & 8.824774 & -0.261117 & 2.473942 \\ \text { 18.C } & 8.958430 & 3.219765 & 2.936125 \\ \text { 19.C } & 10.283223 & 3.603898 & 3.621313 \\ \text { 20.H } & 10.120550 & 3.966790 & 4.639236 \\ \text { 21.H } & 10.730881 & 4.421307 & 3.049001\end{array}$




\begin{tabular}{|c|c|c|c|}
\hline 22.H & 11.008494 & 2.797628 & 3.651694 \\
\hline 23.C & 9.260280 & 2.518424 & 1.615854 \\
\hline 24.H & 9.890832 & 1.640976 & 1.768012 \\
\hline $25 . \mathrm{H}$ & 9.807202 & 3.201273 & 0.956663 \\
\hline $26 . \mathrm{H}$ & 8.360175 & 2.202064 & 1.091643 \\
\hline 27.C & 8.261176 & 4.569184 & 2.711261 \\
\hline $28 . \mathrm{H}$ & 7.309525 & 4.503291 & 2.197060 \\
\hline 29.H & 8.922280 & 5.191144 & 2.099882 \\
\hline $30 . \mathrm{H}$ & 8.106169 & 5.085750 & 3.661261 \\
\hline 31.C & 4.216316 & 1.285303 & 7.397620 \\
\hline $32 . \mathrm{H}$ & 3.175304 & 1.526645 & 7.166788 \\
\hline $33 . \mathrm{C}$ & 4.450256 & -0.207517 & 7.197695 \\
\hline $34 . \mathrm{H}$ & 4.201473 & -0.552375 & 6.197279 \\
\hline 35.H & 3.825667 & -0.762821 & 7.903329 \\
\hline 36.H & 5.489942 & -0.470007 & 7.407239 \\
\hline $37 . C$ & 4.507773 & 1.614520 & 8.864446 \\
\hline 38.H & 5.539373 & 1.354944 & 9.116723 \\
\hline 39.H & 3.849659 & 1.022822 & 9.506042 \\
\hline $40 . \mathrm{H}$ & 4.356613 & 2.662987 & 9.114608 \\
\hline 41.C & 4.706871 & 4.111864 & 6.693277 \\
\hline $42 . \mathrm{H}$ & 4.794674 & 4.573146 & 5.707596 \\
\hline 43.C & 3.260295 & 4.270853 & 7.146970 \\
\hline 44.H & 2.559730 & 3.730875 & 6.511524 \\
\hline $45 . \mathrm{H}$ & 2.987710 & 5.329524 & 7.109616 \\
\hline 46.H & 3.108821 & 3.939257 & 8.174771 \\
\hline 47.C & 5.670599 & 4.852066 & 7.620000 \\
\hline $48 . \mathrm{H}$ & 5.667873 & 4.433811 & 8.629278 \\
\hline 49.H & 5.381033 & 5.903613 & 7.699698 \\
\hline
\end{tabular}




\begin{tabular}{llll} 
50.H & 6.694835 & 4.804583 & 7.246231 \\
51.B & 5.264630 & 0.594513 & 3.909630 \\
52.H & 5.721560 & -0.246602 & 4.592083 \\
53.B & 5.234836 & 3.330471 & 3.475593 \\
54.H & 5.711355 & 4.335918 & 3.869496 \\
55.B & 5.705090 & 2.561386 & 1.942716 \\
56.H & 6.488087 & 3.078895 & 1.233131 \\
57.B & 5.726789 & 0.834653 & 2.216621 \\
58.H & 6.548188 & 0.142345 & 1.728114 \\
59.B & 3.575356 & 1.130405 & 4.097723 \\
60.H & 2.907543 & 0.633447 & 4.937838 \\
\hline 61.B & 3.558417 & 2.862199 & 3.810462 \\
62.H & 2.892008 & 3.603489 & 4.444692 \\
63.B & 4.060883 & 3.158414 & 2.153419 \\
64.H & 3.686939 & 4.117567 & 1.563296 \\
65.B & 4.351289 & 1.577750 & 1.386874 \\
66.H & 4.192378 & 1.392754 & 0.225104 \\
67.B & 4.084395 & 0.321733 & 2.613479 \\
68.H & 3.718972 & -0.779752 & 2.365761 \\
60.H & 3.030496 & 1.756607 & 2.535447 \\
\hline & 1.885289 & 1.694491 & 2.230116 \\
\hline
\end{tabular}




\section{References}

(1) Kivekäs, R.; Sillanpää, R.; Teixidor, F.; Viñas, C.; Nuñez, R.; Abad, M. 1Diisopropylphosphino-2-Methyl-1,2-Dicarba-Closo-Dodecaborane(12), (1), and 1,2Bis(Diisopropylphosphino)-1,2-Dicarba-Closo-Dodecaborane(12), (2), at 193 K. Acta Cryst C 1995, 51, 1864-1868. https://doi.org/10.1107/S0108270195002629.

(2) te Velde, G.; Bickelhaupt, F. M.; Baerends, E. J.; Fonseca Guerra, C.; van Gisbergen, S. J. A.; Snijders, J. G.; Ziegler, T. Chemistry with ADF. J. Comput. Chem. 2001, 22, 931-967. https://doi.org/10.1002/jcc.1056. 